\title{
An improved general kinetic analysis of non-linear irreversible polymerisations
}

\author{
Mário Rui P.F.N. Costa ${ }^{\mathrm{a}, *}$, Rolando C.S. Dias ${ }^{\mathrm{b}}$ \\ ${ }^{a}$ LSRE - Fac. Eng. Univ. Porto, Departamento de Eng. Química, R. Roberto Frias, 4200-465 Porto Codex, Portugal \\ ${ }^{\mathrm{b}}$ LSRE - Instituto Politécnico de Bragança, Quinta de S. Apolónia, 5300 Bragança, Portugal
}

\begin{abstract}
A method to predict average molecular weights before and after gelation for general irreversible non-linear polymerisations forming tree-like molecules is described. Recently developed numerical methods for solving two point boundary value problems are essential for the success of these calculations after gelation and open the way to eventually be able to efficiently predicting chain length distributions. Anionic and free-radical polymerisation of vinyl monomers in the presence of divinyl monomers or with transfer to polymer are taken as case studies. Comparison to experimental data and with simulation results obtained through "numerical fractionation" confirms the usefulness of current approach.
\end{abstract}

Keywords: Polymerisation; Kinetics; Modelling; Gels; Free radical; Anionic

\section{Introduction}

Since more than one decade a few modelling schemes of general polymerisation reactions and reactors have been proposed. Following Villermaux and Blavier (1984), their development has been pursued by, among others, Ray and collaborators (Ray, 1991; Saldivar and Ray, 1995) and Kiparissides and collaborators (Achilias and Kiparissides, 1992; Konstadinides et al., 1992). Fairly general kinds of polymerisations (radical copolymerisations, linear polycondensations and polyadditions ...) have been treated using kinetic approaches, with the aim of giving to engineers and scientists a faster and less error-prone way of dealing with the complex systems which occur in practice. Commercial software packages have been using these principles since more than one decade.

The kinetic approach for modelling irreversible non-linear polymerisations which has been independently developed

\footnotetext{
* Corresponding author. Fax: +351-225-081-666.

E-mail addresses: mrcosta@fe.up.pt (M.R.P.F.N. Costa), rdias@ipb.pt (R.C.S. Dias).
}

by the present authors (Costa and Dias, 1994) shares similar objectives with the above cited lines of thought and is also based in the numerical solution of species conservation equations, but it tries in addition to:

- deal with more general classes of irreversible polymerisations;

- eliminate unwanted assumptions such as the pseudosteady state hypothesis, neglect of multi-radicals or multiple growth centres in general and small ring formation;

- take into account the presence of gel;

- provide ways of efficiently computing molecular weight distributions, molecular average radius of gyration and gel properties.

Its two more distinctive peculiarities reside in the simultaneous use of a description based on sets of stoichiometric coefficients and numerical computation of moment generating functions. It is the combination of the two which makes the originality and strength of this approach: isolated uses of either technique were previously known and are more widespread. 
There is a problem with the easiness of use of the previously defined stoichiometric relations with polyadditions. Therefore, a thorough revision of its basic notation has been carried out in order to improve its numerical performance and applicability to other polymerisation schemes, a task which has been started in a previous work (Costa and Dias, 1995). Prediction of gel properties related to elastic properties and the prediction of $z$-average molecular radius of gyration of polymer molecules (Costa and Dias, 1998) will not here be described in order to keep this presentation within a manageable size.

The whole approach would be close to useless should the equations for the moment generating functions become intractable. Numerical solution of non-linear free radical polymerisation models do present severe problems, which only recently (Costa and Dias, 2003) could be overcome. A general procedure for solving the resulting equations should therefore be considered an essential component of this method in order to make possible its widespread use.

Numerical inversion of moment generating functions of discrete probability distributions or $z$-transforms has also been known (Mills, 1986; Costa and Villermaux, 1988) to be useful for obtaining chain length distributions (CLD) with polycondensations and some non-radical polyadditions (Costa and Dias, 1995). An analysis of its truncation and round-off errors (Abate and Whitt, 1992) shows this is a reliable method. Still, when average chain length increases, it becomes too slow, as it requires as many values of the transform or generating function as the upper value of chain length where distribution is to be evaluated. A better algorithm is needed to deal with that situation. It has been suggested (Abate and Whitt, 1992) that determination of the asymptotic behaviour of the distribution should be tried. This would require finding the singularities of the generating function closer to the origin, or Hayman's method (Hayman, 1956) in case they do not exist. A practical way to do it with numerically estimated generating functions is yet to be found.

In recent years, some researchers have developed the use of Laplace transforms, the continuous counterpart of discrete moment generating functions or $z$-transforms, aiming at computing CLD. After Miller et al. (1996) have shown the potential of this method, Sarmoria and collaborators have been using it in process modelling of polyolefines viscosity breaking by peroxides (Asteasuain et al., 2002a) and both linear (Asteasuain et al., 2004) and branched (Brandolin et al., 2002) simple free radical polymerisations.

There is some convergence of the above described approach with the methods described in this paper, but considerable differences in the mathematical treatments are also conspicuous: a continuous variable approximation is introduced, and partial differential equations are solved using discretisation in dummy Laplace variables, avoiding the method of characteristics. No way to compute CLD in the presence of gel is presented either.
A thorough comparison of the performances of these approaches is certainly worth doing, but is outside the scope of this paper.

Whatever the detailed mathematical approach is used, manipulation of mass balance equations of polymer species is a tedious and error-prone task. The same way as it is possible now to solve fairly complex mathematical problems using commercial packages or open-source programs like Octave, it would be interesting to develop some sort of interpreter of polymerisation schemes. It is hoped that the methods here described can pave the way to such a development.

\section{Statistical description of polymer molecular structure}

\subsection{Nomenclature of groups}

Chemical groups in polymer molecules, as well as monomers, initiators, transfer agents, by-products of polymerisation (such as water liberated in polyesterification reactions), and all other non-polymer molecules, are given the common name $A_{n}$, with $n=1, N_{A}$. Their mole concentration will also be called $A_{n}$. A subset of these comprises the $N_{Y}$ monomers $Y_{1} \ldots Y_{N_{Y}}$.

For each of the monomers there is a corresponding repeating unit (RU) $X_{1} \ldots X_{N_{Y}}$.

Each RU is supposed to contain some root group (RG), to which all other groups in polymer belonging to same RU are directly attached. Root groups are not changed by chemical reaction.

So, degree of polymerisation is the count of RG per molecule.

Besides RG, other examples of polymer groups include the repeating units and any side or main chain groups formed by chemical reaction. A total of $N_{P}$ groups comprises the chemical moieties belonging to polymer molecules.

A sub-set of those $N_{P}$ groups, the active $N_{A_{P}}$ polymer groups, are consumed by chemical reaction.

This notation provides the means of adding new groups and reactions to a polymerisation scheme without disrupting the results previously obtained with a scheme with less groups and reactions.

Our first examples (case studies I and II) are based upon the $n$-butyllithium (or any monofunctional lithium alkyl or aryl) initiated anionic copolymerisation of styrene $\left(Y_{1}\right)$ and $m$ - or $p$-divinylbenzene $\left(Y_{2}\right)$ with ethylbenzene $(S)$ as the solvent.

After a few fundamental studies on its chemical kinetics (Worsfold, 1970; Eschwey and Burchard, 1975) have been carried out, this system was later found to be useful as a model for RIM encapsulation of electronic parts (Your et al., 1989; Christiansen et al., 1990; Karles et al., 1991).

The initiator and the $n$-butyl fragment are groups $A_{1}$ and $A_{2}$ (see Table 3). Styrene monomer $\left(Y_{1}\right.$ or $\left.A_{3}\right)$ is associated to a root group $X_{1}$ (or $A_{4}$ ), which can be identified as the pending phenyl group. 
The 1,1,2-ethanetriyl carbanion with its lithium countercation is an active group belonging to polymer $\left(A_{5}\right)$, while the ethylene group formed after propagation is an example of an inactive group in polymer $\left(A_{6}\right)$.

Copolymerisation with $m$ - or $p$-divinylbenzene $\left(Y_{2}\right)$ introduces the divinyl monomer as a new chemical group not belonging to polymer $\left(A_{7}\right)$. It is associated to a new root group $\left(A_{8}\right)$, a phenylene group.

For $p$-divinylbenzene, kinetic measurements (Worsfold, 1970; Karles et al., 1991) show that the pending vinyl group is about 10 times less reactive than the initial one, and about as reactive as the vinyl groups in styrene and $m$ divinylbenzene. On the contrary, anion reactivities look more or less similar.

So, in case study I, the divinyl monomer will be the meta isomer, so that its lithium anion can be lumped with the lithium anion of styrene into a single anion group $A_{5}$. The same assumptions were used in Flory's classical analysis of non-linear polyadditions (Flory, 1953).

Another active polymer group is the pendant vinyl group A9.

This "ideal" living anionic homopolymerisation styrene/mdivinylbenzene would therefore be described by a set of $N_{A}=9$ groups, from which $N_{P}=6$ belong to polymer ("groups" 1,3 and 7 are chemical compounds, the initiator and monomers styrene and $m$-divinylbenzene). Two active polymer group exist $\left(A_{5}\right.$ and $\left.A_{9}\right)$ and so $N_{A_{P}}=2$.

In case study II, lithium 1,1,2-ethanetriyl anion is a new active group in polymer $A_{10}$. A few secondary reactions (transfer to solvent, termination and transfer through hydride elimination) are also taken into account. The overall number of groups increases now to $N_{A}=18, N_{P}=$ 12 of them belonging to polymer, of which $N_{A_{P}}=5$ are active.

In case study III, free radical copolymerisation of a monovinyl monomer with a divinyl monomer is considered. Styrene copolymerisations with $m$ - and $p$-divinylbenzene, as well as ethylene dimethacrylate and diisopropenylbenzene have been extensively investigated before and after gelation (Hild and Okasha, 1985a,b; Hild et al., 1985) and will be here briefly discussed.

Case study IV is the free radical polymerisation of a single vinyl monomer with transfer to polymer in a batch reactor. This is a classical problem in non-linear free radical polymerisations (Bamford and Tompa, 1954) which has been analysed in the past by the method of the moments (Tobita and Hamielec, 1988) and "numerical fractionation" (Teymour and Campbell, 1994).

\subsection{Molecular chain length and mass distributions}

The vector with $N_{A}$ components containing the counts of groups $A_{1} \ldots A_{N_{A}}$ in a given polymer molecule $P_{m}$, with $m$ being some arbitrary index, will be named $\mathbf{a}_{m}$.
The $N_{A}-N_{P}$ components $a_{j}$ such that $\delta_{P_{j}}=0$ will always be nil and their storage should be avoided in a computational implementation.

The ensemble of molecular trees with same vectors of numbers of groups will be named $P\left(a_{m}\right)$, with mole concentration written as $P\left(\mathbf{a}_{m}\right)$. Letting the counting variable $m$ sweep all natural numbers, an overall mole concentration $P(\mathbf{a})$, is obtained.

$P(\mathbf{a}) / P$ is also the probability function of the number chain length distribution (NCLD) of polymer molecules. We will rather use the mole concentration distribution without normalising by $P$.

Most of the times its vectorial moment generating function $G(\mathbf{s})$ instead of its real counterpart will be used:

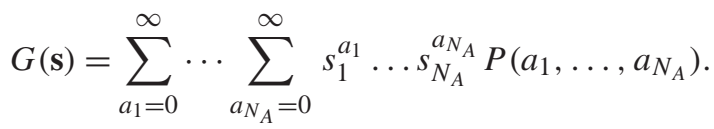

As the $N_{A}-N_{P}$ components $s_{j}$ such that $\delta_{P_{j}}=0$ are useless, they will be considered equal to 1 . The molecular mass $M_{m}$ of a generic molecule $P\left(\mathbf{a}_{m}\right)$ is obtained from the molecular masses of the groups it contains:

$M_{m}=\sum_{j=1}^{N_{A}} a_{j_{m}} M_{A_{j m}}$.

Number molecular mass distribution (NMMD) of polymer molecules is related to the NCLD by introducing its moment generating function, as shown in Table 1. Some widely used average molecular masses are defined as the ratios of integer moments relative to molecular mass, obtained by differentiation of $G$, and are thus related to the moments relative to the numbers of groups.

A convenient notation for those moments adapts the index convention for partial derivatives, leading to expressions like those below, using the abbreviation $\mathbf{1}_{N}$ for a vector of $N$ components all equal to 1 :

$$
\begin{aligned}
\lambda_{n \ldots p} & =\sum_{a_{1}=0}^{\infty} \ldots \sum_{a_{N_{A}}=0}^{\infty} a_{n} \ldots a_{p} P(\mathbf{a}) \\
& =\frac{\partial \ldots \partial G}{\partial \log s_{n} \ldots \partial \log s_{p}}\left(\mathbf{1}_{N_{A}}\right) \\
& =G_{n \ldots p}\left(\mathbf{1}_{N_{A}}\right)
\end{aligned}
$$

Oth order moment is simply $\lambda_{0}$. Moments with any index $j$ such that $\delta_{P_{j}}=0$ are obviously nil.

Well-known definitions of number, weight and $z$-average molecular masses make use of lower integer moments of NMMD, and can thus be computed as further shown in Table 1.

\subsection{Kinetic description of polymer formation}

A classification of chemical reactions will be next introduced according to their effects on the number and kind of 
Table 1

Summary of notation describing molecular mass distributions

\begin{tabular}{|c|c|}
\hline Variable & Mathematical definition \\
\hline Moment generating function of number molecular mass distribution (NMMD) & $G\left(s_{M}\right)=\sum_{m=1}^{\infty} s_{M}^{M_{m}} P\left(M_{m}\right)=G\left(s_{M}^{M_{A_{1}}}, \ldots, s_{M}^{M_{A_{N}}}\right)$ \\
\hline First-order moment of NMMD & $\lambda_{M}=\sum_{n=1}^{N_{A}} M_{A_{n}} \lambda_{n}$ \\
\hline Second-order moment of NMMD & $\lambda_{M M}=\sum_{m=1}^{N_{A}} \sum_{n=1}^{N_{A}} M_{A_{m}} M_{A_{n}} \lambda_{m n}$ \\
\hline Third-order moment of NMMD & $\lambda_{M M M}=\sum_{l=1}^{N_{A}} \sum_{m=1}^{N_{A}} \sum_{n=1}^{N_{A}} M_{A_{l}} M_{A_{m}} M_{A_{n}} \lambda_{l m n}$ \\
\hline Number-average molecular mass & $\bar{M}_{n}=\frac{\lambda_{M}}{\lambda_{0}}$ \\
\hline Weight-average molecular mass & $\bar{M}_{w}=\frac{\lambda_{M M}}{\lambda_{M}}$ \\
\hline$z$-average molecular mass & $\bar{M}_{z}=\frac{\lambda_{M M M}}{\lambda_{M M}}$ \\
\hline
\end{tabular}

Table 2

Summary of notation describing polymerisation reactions

\begin{tabular}{|c|c|c|c|}
\hline Reaction description & Number & Rate constant & Net change of number of polymer molecules \\
\hline $\begin{array}{l}\text { Polymer/polymer propagations, } \\
\text { condensations or terminations }\end{array}$ & $N_{R_{P}}$ & $\begin{array}{l}k_{n} \\
n=1, N_{R_{P}}\end{array}$ & -1 \\
\hline Polymer/monomer propagations & $N_{R}-N_{R_{P}}$ & $\begin{array}{l}k_{n} \\
n=N_{R_{P}}+1, N_{R}\end{array}$ & 0 \\
\hline Unimolecular reactions of polymer & $N_{R_{P}}^{*}$ & $\begin{array}{l}k_{n}^{*} \\
n=1, N_{R_{P}}^{*}\end{array}$ & 0 \\
\hline Unimolecular reactions & $N_{R}^{*}-N_{R_{P}}^{*}$ & $\begin{array}{l}k_{n}^{*} \\
n=N_{R_{P}}^{*}+1, N_{R}^{*}\end{array}$ & 0 \\
\hline $\begin{array}{l}\text { Polymer/polymer reactions, no new } \\
\text { connections between RU }\end{array}$ & $N_{R_{P}}^{* *}$ & $\begin{array}{l}k_{n}^{* *} \\
n=1, N_{R_{P}}^{* *}\end{array}$ & 0 \\
\hline $\begin{array}{l}\text { Polymer/non-polymer reactions, no } \\
\text { new connections between RU }\end{array}$ & $N_{R_{S}}^{* *}$ & $\begin{array}{l}k_{n}^{* *} \\
n=N_{R_{P}}^{* *}+1, N_{R_{P}}^{* *}+N_{R_{S}}^{* *}\end{array}$ & 0 \\
\hline $\begin{array}{l}\text { Non-polymer/non-polymer reactions, } \\
\text { without creation of new polymer } \\
\text { molecules }\end{array}$ & $N_{S}$ & $\begin{array}{l}k_{n}^{* *} \\
n=N_{R_{P}}^{* *}\end{array}+N_{R_{S}}^{* *}+1, N_{R}^{* *}$ & 0 \\
\hline Bimolecular initiations & $N_{I}$ & $\begin{array}{l}k_{I_{n}} \\
n=1, N_{I}\end{array}$ & 1 \\
\hline Transfers to monomers & $N_{M}$ & $\begin{array}{l}k_{M_{n}} \\
n=1, N_{M}\end{array}$ & 1 \\
\hline
\end{tabular}

polymer molecules. Table 2 summarises the notations here used. Some reactions (propagation, termination or end group linking) create connections between repeating units and thus lead to an increase of molecular mass. A total of $N_{R}$ such reactions are supposed to create connection between RU.

First $N_{R_{P}}$ reactions of this class are supposed to involve only groups present in polymer molecules. So, they cause the net disappearance of a polymer molecule.
The other $N_{R}-N_{R_{P}}$ reactions occur between a group belonging to a polymer molecule and a non-polymeric molecule. Therefore, the overall number of polymer molecules is conserved. Tables 3 and 4 summarize the descriptions of groups in cases I and II respectively.

In case study I, $N_{R_{P}}=1$ (propagation over pending double bonds in polymer), while another 2 involve a monomer and a polymer anion (Table 5); in case study II, as reactivities of 
Table 3

Description of groups in a simplified model of anionic copolymerisation of styrene with $m$-divinylbenzene (case study I)

\begin{tabular}{|c|c|c|c|c|c|c|}
\hline Group description & $n$ & $\delta_{P_{j}}$ & $\delta_{A_{j}}$ & Alias & Chemical formula & $M_{A_{n}}$ \\
\hline$n$-butyllithium & 1 & 0 & 0 & $I$ & $\mathrm{LiC}_{4} \mathrm{H}_{9}$ & 64 \\
\hline Initiator fragment & 2 & 1 & 0 & & $-\mathrm{C}_{4} \mathrm{H}_{9}$ & 57 \\
\hline Styrene & 3 & 0 & 0 & $Y_{1}$ & $\mathrm{CH}_{2}=\mathrm{CHC}_{6} \mathrm{H}_{5}$ & 104 \\
\hline Phenyl in RG styryl & 4 & 1 & 0 & $X_{1}$ & $-\mathrm{C}_{6} \mathrm{H}_{5}$ & 77 \\
\hline Lithium carbanion & 5 & 1 & 1 & & $-\mathrm{CH}_{2} \mathrm{CH}^{-}(\ldots) \mathrm{Li}^{+}$ & 34 \\
\hline 1,1,2-ethanetriyl & 6 & 1 & 0 & & $-\mathrm{CH}_{2} \mathrm{CH}<$ & 27 \\
\hline m-divinylbenzene (DVB) & 7 & 0 & 0 & $Y_{2}$ & $\left(\mathrm{CH}_{2}=\mathrm{CH}\right)_{2} \mathrm{C}_{6} \mathrm{H}_{4}$ & 130 \\
\hline Phenylene in RG from DVB & 8 & 1 & 0 & $X_{2}$ & $-\mathrm{C}_{6} \mathrm{H}_{4}-$ & 76 \\
\hline Pendant vinyl group in polymer & 9 & 1 & 1 & & $-\mathrm{CH}=\mathrm{CH}_{2}$ & 27 \\
\hline
\end{tabular}

Table 4

Description of groups in anionic copolymerisation of styrene with a divinylbenzene (case study II)

\begin{tabular}{|c|c|c|c|c|c|c|}
\hline Group description & $n$ & $\delta_{P_{j}}$ & $\delta_{A_{j}}$ & Alias & Chemical formula & $M_{A_{n}}$ \\
\hline$n$-butyllithium & 1 & 0 & 0 & $I$ & $\mathrm{LiC}_{4} \mathrm{H}_{9}$ & 64 \\
\hline Initiator fragment & 2 & 1 & 0 & & $-\mathrm{C}_{4} \mathrm{H}_{9}$ & 57 \\
\hline Styrene & 3 & 0 & 0 & $Y_{1}$ & $\mathrm{CH}_{2}=\mathrm{CHC}_{6} \mathrm{H}_{5}$ & 104 \\
\hline Phenyl in RG styryl & 4 & 1 & 0 & $X_{1}$ & $-\mathrm{C}_{6} \mathrm{H}_{5}$ & 77 \\
\hline Lithium carbanion from styrene & 5 & 1 & 1 & & $-\mathrm{CH}_{2} \mathrm{CH}^{-}(\ldots) \mathrm{Li}^{+}$ & 34 \\
\hline 1,1,2-ethanetriyl & 6 & 1 & 0 & & $-\mathrm{CH}_{2} \mathrm{CH}<$ & 27 \\
\hline$p$-divinylbenzene (DVB) & 7 & 0 & 0 & $Y_{2}$ & $\left(\mathrm{CH}_{2}=\mathrm{CH}\right)_{2} \mathrm{C}_{6} \mathrm{H}_{4}$ & 130 \\
\hline Phenylene in RG from DVB & 8 & 1 & 0 & $X_{2}$ & $-\mathrm{C}_{6} \mathrm{H}_{4}-$ & 76 \\
\hline Pendant vinyl group in polymer & 9 & 1 & 1 & & $-\mathrm{CH}=\mathrm{CH}_{2}$ & 27 \\
\hline Lithium carbanion from DVB & 10 & 1 & 1 & & $-\mathrm{CH}_{2} \mathrm{CH}^{-}(\ldots-) \mathrm{Li}^{+}$ & 34 \\
\hline Terminal ethylene & 11 & 1 & 0 & & $-\mathrm{CH}_{2} \mathrm{CH}_{2}(\ldots)$ & 28 \\
\hline \multicolumn{7}{|c|}{ Vinylene anion (one hydrogen is considered to } \\
\hline belong to the contiguous repeating unit) & 12 & 1 & 0 & & $-\mathrm{CH}^{-} \mathrm{Li}^{+}=\mathrm{CH}(\ldots)$ & 32 \\
\hline Terminal vinylene from styrene & 13 & 1 & 1 & & $-\mathrm{CH}=\mathrm{CH}-(\ldots)$ & 26 \\
\hline Terminal vinylene from DVB & 14 & 1 & 1 & & $-\mathrm{CH}=\mathrm{CH}-(\ldots-)$ & 26 \\
\hline Lithium hydride & 15 & 0 & 0 & & $\mathrm{LiH}$ & 8 \\
\hline Ethylbenzene (EB) & 16 & 0 & 1 & $S$ & $\mathrm{CH}_{3} \mathrm{CH}_{2} \mathrm{C}_{6} \mathrm{H}_{5}$ & 106 \\
\hline Lithium carbanion from EB & 17 & 0 & 1 & & $\mathrm{CH}^{-}\left(\mathrm{CH}_{3}\right) \mathrm{C}_{6} \mathrm{H}_{5} \mathrm{Li}^{+}$ & 112 \\
\hline Phenylmethylmethine end group & 18 & 1 & 0 & & $-\mathrm{CH}\left(\mathrm{CH}_{3}\right) \mathrm{C}_{6} \mathrm{H}_{5}$ & 105 \\
\hline
\end{tabular}

Table 5

Reaction stoichiometry for a simplified model of anionic copolymerisation of styrene and a divinylbenzene (case study I)

\begin{tabular}{|c|c|c|}
\hline Reaction name & Chemical equation & Stoichiometric functions \\
\hline Styrene initiation & $I+Y_{1} \stackrel{k_{I_{1}}}{\longrightarrow} A_{2}+X_{1}+A_{5}$ & $\Psi_{I_{1}}=s_{2} s_{4} s_{5}$ \\
\hline DVB initiation & $I+Y_{2} \stackrel{k_{I}}{\longrightarrow} A_{2}+X_{2}+A_{5}+A_{9}$ & $\Psi_{I_{2}}=s_{2} s_{5} s_{8} s_{9}$ \\
\hline Pendant vinyl initiation & $I+A_{9} \stackrel{k_{1}^{* *}=k_{I_{3}}}{\longrightarrow} A_{2}+A_{5}$ & $\begin{array}{l}\Psi_{1}^{* *-}=1 \\
\Psi_{1}^{* *+}=\frac{s_{2} s_{5}}{s_{9}}\end{array}$ \\
\hline Propagation on pendant vinyl group & $A_{5}+A_{9} \stackrel{k_{1}=k_{p}}{\longrightarrow} A_{6}+A_{5}$ & $\begin{array}{l}\Psi_{1}^{-}=\frac{s_{6}}{s_{5}} \\
\Psi_{1}^{+}=\frac{s_{5}}{s_{9}}\end{array}$ \\
\hline Propagation on styrene & $Y_{1}+A_{5} \stackrel{k_{2}=k_{p}}{\longrightarrow} A_{5}+X_{1}+A_{6}$ & $\begin{array}{l}\Psi_{2}^{-}=s_{4} s_{5} \\
\Psi_{2}^{+}=\frac{s_{6}}{s_{5}}\end{array}$ \\
\hline Propagationon DVB & $A_{5}+Y_{2} \stackrel{k_{3}=k_{p}}{\longrightarrow} X_{2}+A_{5}+A_{9}+A_{6}$ & $\begin{array}{l}\Psi_{3}^{-}=\frac{s_{6}}{s_{5}} \\
\Psi_{3}^{+}=s_{5} s_{8} s_{9}\end{array}$ \\
\hline
\end{tabular}

carbanions and double bonds are distinguished according to the nature of the repeating unit or monomer to which they are attached, 6 different propagation reactions are defined (Table 6).

The $n$th such reaction involves the groups $A_{g_{n}^{-}}$and $A_{g_{n}^{+}}$. It will be assumed that $g_{n}^{-} \leqslant g_{n}^{+}$.
In order to avoid the multiple levels of indexing as above and make the resulting expressions more readable, a notation inspired in the way computer assembly languages describe indirect addressing will be used, as vectors $\mathbf{g}^{+}$and $\mathbf{g}^{-}$are just collections of indices. Thus, the two groups directly involved in the $n$th reaction 
Table 6

Reaction stoichiometry of anionic copolymerisation of styrene and a divinylbenzene (case study II)

\begin{tabular}{|c|c|c|}
\hline Reaction name & Chemical equation & Stoichiometric functions \\
\hline Styrene initiation & $I+Y_{1} \underset{k_{l}}{\stackrel{k_{I_{1}}}{\rightarrow}} A_{2}+X_{1}+A_{5}$ & $\Psi_{I_{1}}=s_{2} s_{4} s_{5}$ \\
\hline DVB initiation & $I+Y_{2} \stackrel{{ }^{K} I_{2}}{\longrightarrow} A_{2}+X_{2}+A_{9}+A_{10}$ & $\Psi_{I_{2}}=s_{2} s_{8} s_{9} s_{10}$ \\
\hline Pendant vinyl initiation & $I+A_{9} \stackrel{k_{1}^{* *}=k_{I}}{\longrightarrow} A_{2}+A_{10}$ & $\begin{array}{l}\Psi_{1}^{* *-}=1 \\
\Psi_{1}^{* *+}=\frac{s_{2} s_{10}}{s_{9}} \\
\Psi^{-}=\underline{s_{6}}\end{array}$ \\
\hline Propagation of styryl anion on pendant vinyl group & $A_{5}+A_{9} \stackrel{k_{1}=k_{p}}{\longrightarrow} A_{6}+A_{10}$ & $\begin{array}{l}\Psi_{1}^{-}=\frac{s_{6}}{s_{5}} \\
\Psi_{1}^{+}=\frac{s_{10}}{s_{9}}\end{array}$ \\
\hline Propagation of DVB anion on pendant vinyl group & $A_{9}+A_{10} \stackrel{k_{2}=k_{p_{23}}}{\longrightarrow} A_{6}+A_{10}$ & $\begin{array}{l}\Psi_{2}^{-}=\frac{s_{10}}{s_{9}} \\
\Psi_{2}^{+}=\frac{s_{6}}{s_{10}} \\
\Psi^{-}=S_{5} S_{5}\end{array}$ \\
\hline Propagation of styryl anion on styrene & $Y_{1}+A_{5} \stackrel{k_{3}=k_{p_{11}}}{\longrightarrow} A_{5}+X_{1}+A_{6}$ & $\begin{array}{l}\Psi_{3}^{-}=s_{4} s_{5} \\
\Psi_{3}^{+}=\frac{s_{6}}{s_{5}}\end{array}$ \\
\hline Propagation of DVB anion on styrene & $Y_{1}+A_{10} \stackrel{k_{4}=k_{p_{21}}}{\longrightarrow} A_{5}+X_{1}+A_{6}$ & $\begin{array}{l}\Psi_{4}^{-}=s_{4} s_{5} \\
\Psi_{4}^{+}=\frac{s_{6}}{s_{5}}\end{array}$ \\
\hline Propagation of styryl anion on DVB & $A_{5}+Y_{2} \stackrel{k_{5}=k_{p_{12}}}{\longrightarrow} X_{2}+A_{9}+A_{10}+A_{6}$ & $\begin{array}{l}\Psi_{5}^{-}=\frac{s_{6}}{s_{5}} \\
\Psi_{5}^{+}=s_{8} s_{9} s_{10}\end{array}$ \\
\hline Propagation of DVB anion on DVB & $Y_{2}+A_{10} \stackrel{k_{6}=k_{p_{22}}}{\longrightarrow} X_{2}+A_{9}+A_{10}+A_{6}$ & $\begin{array}{l}\Psi_{6}^{-}=s_{8} s_{9} s_{10} \\
\Psi_{6}^{+}=\frac{s_{6}}{s_{10}}\end{array}$ \\
\hline
\end{tabular}

coalescing polymer molecules will rather be designated as $A_{[n-]}$ and $A_{[n+]}$.

The other groups in the same repeating unit may also be transformed by that reaction (first shell substitution effect). By introducing stoichiometric coefficients, equal to the numbers of moles in products minus the number of moles in reagents for each group (or species), taking the formation of 1 mole of bonds as reference, the $N_{R}$ bimolecular reactions between groups $A_{[n-]}$ and $A_{[n+]}$ are written as:

$\sum_{j=1}^{N_{A}}\left(v_{n j}^{-}+v_{n j}^{+}\right) A_{j}=0, \quad n=1, N_{R}$.

Two sets of stoichiometric coefficients have been distinguished: those which concern the groups connected to the $\mathrm{RU}$ at the RG attached to group, and those related to the other RG. They may be equal in the less frequent cases where an end-group reacts with itself, such as in the condensation of silanols.

The rate of the $n$th reaction of this kind will be written as:

$R_{n}=k_{n} A_{[n-]} A_{[n+]}$.

According to Flory's Principle of Equal Reactivity, $k_{n}$ does not depend on the nature of the molecule to which the groups are attached, but the above defined pseudo second-order rate constant $k_{n}$ is often not a true constant and is a function of the composition of the reaction media.

This remark is particularly pertinent in anionic polymerisation of hydrocarbons (Table 5). The observed fractional orders with respect to initiator, both in propagation and initiation, have been described through a simple association model for anionic species (Lewis and Brown, 1970; Bywater and Worsfold, 1967; Bywater, 1998). Only a fraction of the anions is available at every moment for participating in initiation or propagation. That fraction should be the same for every chemically similar anionic group, irrespective of molecular chain length (Worsfold and Bywater, 1960; Arest-Yakubovich, 1997). Apparent kinetic constants should follow a power law with respect to overall anion concentration, with a negative exponent slightly less than 1, explaining thus the observed apparent orders with respect to initiator which are much less than 1 .

In general, $N_{I}$ bimolecular initiation reactions involving a pair of groups $A_{[I n-]}$ and $A_{[I n+]}$ not present in polymer, with rate constants $k_{I_{n}}$, create a new polymer molecule with a vector of groups $v_{I_{n}}$ :

$A_{[I n-]}+A_{[I n+]} \stackrel{k_{I_{n}}}{\longrightarrow} \mathrm{P}\left(v_{I_{n}}\right)$.

There are in case study II six initiation reactions (see Table 6), four of them creating new polymer molecules from non-polymeric ones; the other two are the initiations of the pendent double bonds, either by initiator or by an anion formed by transfer to solvent.

Other reactions between groups, both bimolecular (for example, termination by dismutation in free-radical polymerisation), sometimes unimolecular (such as the termination by hydride expulsion in anionic polymerisation), do not create connections between repeating units, and do not change the number of polymer molecules.

It is assumed there are $N_{R}^{*}$ such unimolecular reactions, $N_{R_{P}}^{*}$ of which involving groups in polymer molecules and the others only non-polymer molecules (for instance, initiator decomposition), with rate constants $k_{n}^{*}$ :

$\sum_{j=1}^{N_{A}} v_{n j}^{*} A_{j}=0, \quad n=1, N_{R}^{*}$ 
Table 7

Reaction stoichiometry of anionic copolymerisation of styrene and a divinylbenzene (case study II) (cont.)

\begin{tabular}{|c|c|c|}
\hline Reaction name & Chemical equation & Stoichiometric functions \\
\hline Hydride elimination of $\mathrm{A}_{5}$ & $A_{5} \stackrel{k_{1}^{*}=k_{e_{1}}}{\longrightarrow} A_{13}+A_{15}$ & $\Psi_{1}^{*}=\frac{s_{13}}{s_{5}}$ \\
\hline Hydride elimination of $A_{10}$ & $A_{10} \stackrel{k_{2}^{*}=k_{e_{2}}}{\longrightarrow} A_{14}+A_{15}$ & $\Psi_{2}^{*}=\frac{s}{14}_{s_{10}}$ \\
\hline Transfer of styryl anion to vinylene group from styrene & $A_{5}+A_{13} \stackrel{k_{2}^{* *}=k_{t_{11}}}{\longrightarrow} A_{11}+A_{12}$ & $\begin{array}{l}\Psi_{2}^{* *-}=\frac{s_{11}}{s_{5}} \\
\Psi_{2}^{* *+}=\frac{s_{12}}{s_{13}}\end{array}$ \\
\hline Transfer of styryl anion to vinylene group from DVB & $A_{5}+A_{14} \stackrel{k_{3}^{* *}=k_{t}}{\longrightarrow} A_{11}+A_{12}$ & $\begin{array}{l}\Psi_{3}^{* *-}=\frac{s_{11}}{s_{5}} \\
\Psi_{3}^{* *+}=\frac{s_{12}}{s_{14}}\end{array}$ \\
\hline Transfer of DVB anion to vinylene group from styrene & $A_{10}+A_{13} \stackrel{k_{4}^{* *}=k_{t_{21}}}{\longrightarrow} A_{11}+A_{12}$ & $\begin{array}{l}\Psi_{4}^{* *-}=\frac{s_{11}}{s_{10}} \\
\Psi_{4}^{* *+}=\frac{s_{12}}{s_{14}}\end{array}$ \\
\hline Transfer of DVB anion to vinylene group from DVB & $A_{10}+A_{14} \stackrel{k_{5}^{* *}=k_{t 22}}{\longrightarrow} A_{11}+A_{12}$ & $\begin{array}{l}\Psi_{5}^{* *-}=\frac{s_{11}}{s_{5}} \\
\Psi_{5}^{* *+}=\frac{s_{12}}{s_{13}}\end{array}$ \\
\hline Transfer of styrene anion to solvent & $A_{5}+S \stackrel{k_{6}^{* *}=k_{S_{1}}}{\longrightarrow} A_{11}+A_{17}$ & $\Psi_{6}^{* *-}=\frac{s_{11}}{s_{5}}$ \\
\hline Transfer of DVB anion to solvent & $A_{10}+S \stackrel{k_{7}^{* *}=k_{S_{2}}}{\longrightarrow} A_{11}+A_{17}$ & $\Psi_{7}^{* *-}=\frac{s_{11}}{s_{10}}$ \\
\hline Initiation of styrene by solvent anion & $Y_{1}+A_{17} \underset{k_{l_{5}}}{\stackrel{k_{I_{4}}}{\longrightarrow}} A_{18}+X_{1}+A_{5}$ & $\Psi_{I_{4}}=s_{18} s_{4} s_{5}$ \\
\hline $\begin{array}{l}\text { Initiation of DVB by solvent anion } \\
\text { Initiation of pendant vinyl by solvent anion }\end{array}$ & $\begin{array}{l}Y_{2}+A_{17} \stackrel{{ }^{K_{5}}}{\longrightarrow} A_{18}+X_{2}+A_{9}+A_{10} \\
A_{9}+A_{17} \stackrel{k_{8}^{* *}=k_{1}}{\longrightarrow} A_{18}+A_{10}\end{array}$ & $\begin{array}{l}\Psi_{I_{4}}=s_{18} s_{8} s_{9} s_{10} \\
\Psi_{8}^{* *-}=\frac{s_{10} s_{18}}{s_{9}} \\
\Psi_{8}^{* *+}=1\end{array}$ \\
\hline
\end{tabular}

Hydride elimination in anionic polymerisations (Kern et al., 1972; Spach et al., 1962) is an example of these reactions. It transforms the carbanions into terminal vinylene groups. Two different elimination reactions can be distinguished in case study II according to the nature of the carbanion, as shown in Table 7.

Intramolecular reactions can be described using this formalism. The pair of groups in same molecule which react intramolecularly have to be defined as a new active group.

$N_{R}^{* *}$ bimolecular reactions between groups in polymer molecules, with rate constants $k_{n}^{* *}$ do not create new connections between repeating units. Transfer reactions are an example. Their description in terms of stoichiometric coefficients follows from the definitions below:

$\sum_{j=1}^{N_{A}}\left(v_{n j}^{* *-}+v_{n j}^{* *+}\right) A_{j}=0, \quad n=1, N_{R}^{* *}$.

The degradative transfer reaction between styryl carbanions and the terminal vinylene groups is also an example of such reactions. It is convenient to distinguish two kinds of reactions among this latter class:

- The first $N_{R_{p}}^{* *}$ involve two polymer molecules, examples being transfer to polymer, termination by dismutation and the transfers from anions to vinylene end groups in anionic polymerisation (case study II);

- The next $N_{R_{S}}^{* *}$ involve one polymer molecule and a small molecule, a typical example being transfer to solvent;

- The remaining $N_{S}$ reactions concern only small molecules.

For $N_{M}$ reactions between a group in a polymer molecule and a monomer (transfer to monomer), with rate constants
$k_{M_{n}}$, a new polymer molecule is created from that monomer:

$\sum_{j=1}^{N_{A}}\left(v_{M_{n j}}^{-}+v_{M_{n j}}^{+}\right) A_{j} \stackrel{k_{M_{n}}}{\longrightarrow} \mathrm{P}\left(v_{M_{n}}^{+}\right), \quad n=1, N_{M}$

Notice that the new polymer molecule with degree of polymerisation one does not keep unchanged the previously extant double bonds in monomer-their reactivity is most often much lower than double bonds of monomer. An important exception is vinyl acetate.

In any case, this model keeps track of such reactivity changes.

The overall rate of formation or disappearance of groups by chemical reaction is the sum of the contributions of the above-mentioned processes:

$$
\begin{aligned}
R_{A_{n}}= & \sum_{m=1}^{N_{R}} k_{m}\left(v_{m n}^{-}+v_{m n}^{+}\right) A_{[m-]} A_{[m+]} \\
& +\sum_{m=1}^{N_{R}^{*}} k_{m}^{*} v_{m n}^{*} A_{[m *]} \\
& +\sum_{m=1}^{N_{R}^{* *}} k_{m}^{* *}\left(v_{m n}^{* *-}+v_{m n}^{* *+}\right) A_{[m * *-]} A_{[m * *+]} \\
& +\sum_{m=1}^{N_{I}} k_{I_{m}} v_{I_{m n}} A_{[I m-]} A_{[I m+]} \\
& +\sum_{m=1}^{N_{M}} k_{M_{m}}\left(v_{M_{m n}^{-}}^{-}+v_{M_{m n}}^{+}\right) A_{[M m-]} A_{[M m+]} .
\end{aligned}
$$

Whenever the excess volume of mixing of the various species can be neglected, the density of the mixture in the reac- 
tor is easily computed from the knowledge of the volume difference between products and reagents for each chemical reaction. Defining $\Delta V_{m}, \Delta V_{m}^{*}$ and $\Delta V_{m}^{* *}$, and so on, as the mole volume changes due to each kind of reaction in the system (because of density changes and evaporation or precipitation of by-products), the rate of relative change of volume caused by chemical reactions, $R_{v}$, results from the expression below:

$$
\begin{aligned}
R_{v}= & \sum_{m=1}^{N_{R}} k_{m} A_{[m-]} A_{[m+]} \Delta V_{m}+\sum_{m=1}^{N_{R}^{*}} k_{m}^{*} A_{[m *]} \Delta V_{m}^{*} \\
& +\sum_{m=1}^{N_{R}^{* *}} k_{m}^{* *} A_{[m * *-]} A_{[m * *+]} \Delta V_{m}^{* *} \\
& +\sum_{m=1}^{N_{I}} k_{I_{m}} A_{[I m-]} A_{[I m+]} \Delta V_{I_{m}} \\
& +\sum_{m=1}^{N_{M}} k_{M_{m}} A_{[M m-]} A_{[M m+]} \Delta V_{M_{m}} .
\end{aligned}
$$

It is also possible to write a rate equation for the overall number of polymer molecules:

$$
\begin{aligned}
R_{\lambda_{0}}= & -\sum_{m=1}^{N_{R_{P}}} k_{m} A_{[m-]} A_{[m+]}+\sum_{m=1}^{N_{I}} k_{I_{m}} A_{[I m-]} A_{[I m+]} \\
& +\sum_{m=1}^{N_{M}} k_{M_{m}} A_{[M m-]} A_{[M m+]} .
\end{aligned}
$$

The following auxiliary functions, extensively used from now on, will be named stoichiometric functions:

$$
\begin{aligned}
& \Psi_{L}^{U}(\mathbf{s})=\prod_{\substack{j=1 \\
\delta_{P_{j}}=1}}^{N_{A}}\left(s_{j}\right)^{v_{L_{j}}^{U}}, \\
& U=+;-; * ; * *+; * *-, \\
& L=n ; I_{n} ; M_{n} .
\end{aligned}
$$

Methods documented in Costa and Dias (1994) make now possible to write the generating function of the rate equations of formation of polymer species by chemical reaction:

$$
\begin{aligned}
G_{R_{P}}= & \sum_{m=1}^{N_{R_{P}}} k_{m}\left(\Psi_{m}^{-} \Psi_{m}^{+} \frac{\partial G}{\partial \log s_{[m-]}} \frac{\partial G}{\partial \log s_{[m+]}}\right. \\
& \left.-\frac{\partial G}{\partial \log s_{[m-]}} A_{[m+]}-\frac{\partial G}{\partial \log s_{[m+]}} A_{[m-]}\right) \\
& +\sum_{m=N_{R_{P}}+1}^{N_{R}} k_{m} \frac{\partial G}{\partial \log s_{[m-]}} A_{[m+]}\left(\Psi_{m}^{-} \Psi_{m}^{+}-1\right) \\
& +\sum_{m=1}^{N_{R_{P}}^{*}} k_{m}^{*} \frac{\partial G}{\partial \log s_{[m *]}}\left(\Psi_{m}^{*}-1\right)
\end{aligned}
$$

$$
\begin{aligned}
& +\sum_{m=1}^{N_{R_{P}}^{* *}} k_{m}^{* *}\left[\frac{\partial G}{\partial \log s_{[m * *-]}} A_{[m * *+]}\left(\Psi_{m}^{* *-}-1\right)\right. \\
& \left.+\frac{\partial G}{\partial \log s_{[m * *+]}} A_{[m * *-]}\left(\Psi_{m}^{* *+}-1\right)\right] \\
& +\sum_{m=N_{R_{P}}^{*}+1}^{N_{R}^{* *}-N_{S}} k_{m}^{* *} \frac{\partial G}{\partial \log s_{[m * *-]}} A_{[m * *+]}\left(\Psi_{m}^{* *-}-1\right) \\
& +\sum_{m=1}^{N_{I}} k_{I_{m}} A_{[I m-]} A_{[I m+]} \Psi_{I_{m}}+\sum_{m=1}^{N_{M}} k_{M_{m}} A_{[M m+]} \\
& \times\left[A_{[M m-]} \Psi_{M_{m}}^{+}+\frac{\partial G}{\partial \log s_{[M m-]}}\left(\Psi_{M_{m}}^{-}-1\right)\right] .
\end{aligned}
$$

\section{Prediction of average molecular weights and chain length distributions in ideal reactors: general aspects}

\subsection{Mass balance equations}

Integration of mass balance equations for a non-steady state perfectly mixed continuous stirred tank reactor will be done in the same way as described in Costa and Dias (1994). For a reaction volume $V$ and inlet and outlet flow-rates $Q_{F}$ and $Q$, the following overall mass balance holds:

$\frac{\mathrm{d} V}{\mathrm{~d} t}=R_{v} V+Q_{F}(t)-Q(t)$.

Introducing a space-time $\tau$ based on the inlet flow-rate, $\tau=$ $V / Q_{F}$, the mass balance of an active group $A_{j}$ becomes:

$\frac{\mathrm{d} A_{j}}{\mathrm{~d} t}=R_{A_{j}}+\frac{A_{j_{F}}-A_{j}}{\tau}-R_{v} A_{j}$,

$A_{j \mid t=0}=A_{j_{0}}$.

Space-time $\tau$ may be an unknown function of time and reaction volume. It can be computed with help of Eq. (15) and suitable additional conditions.

Moment generating functions of the distributions above defined also verify similar mass balance equations, which are first-order partial differential equations. The moment generating function of the NCLD of polymer $G(\mathbf{s})$ is obtained by solving Eq. (18) with the initial condition Eq. (19), simultaneously with the above mass balances of groups Eq. (16) and initial conditions given by Eq. (17):

$$
\begin{aligned}
& \frac{\partial G}{\partial t}=G_{R_{P}}+\frac{G_{F}(t)-G}{\tau}-R_{v} G, \\
& G_{\mid t=0}=G_{0}\left[\mathbf{s}_{0}(t, \mathbf{s})\right] .
\end{aligned}
$$

The solution of similar equations by the method of characteristics (Courant and Hilbert, 1962) has already been presented in detail elsewhere (Costa and Dias, 1994, 2003), 
and so only the final results are shown. The system of characteristic equations for computing the moment generating function of polymer NCLD can be found in Appendix A.

In order to compute $G(\mathbf{s})$ for complex values along a circle or a spherical surface (according to the number of dimensions of the problem) of radius close to 1 centred in the origin (Abate and Whitt, 1992; Mills, 1986; Costa and Villermaux, 1988), as well as its derivatives at $\mathbf{s}=1_{N_{A}}$ (for the evaluation of the moments), it is necessary to compute the starting vector of the characteristics $\mathbf{s}_{0}$ for each value of $\mathbf{s}$. There are two situations which require very different levels of computing effort:

- For the evaluation of average degrees of polymerisation and average molecular masses before gelation, since $\mathbf{s}_{0}=$ $1_{N_{A}}$ it is only necessary to set $\mathbf{s}=1_{N_{A}}$ in Eqs. (A.1)-(A.3) or additional differential equations for time derivatives along characteristics of higher derivatives of $G$, and a set of ordinary differential equations with respect to time is obtained, leading to an initial value problem, which is more or less easily solved. As before stated, it is recommended to always carry out a prediction of gel time by integration of the above-mentioned initial value problem.

- If there is gel, or if $\mathbf{s} \neq 1_{N_{A}}$ (for computing the NCLD), vector $\mathbf{s}_{0}$ must be found, leading to a boundary value problems of the "time-like" variety (Deuflhard and Bornemann, 2002) for obtaining a numerical solution of the boundary value problem for computing characteristics. A shooting method using integration for increasing time values is the natural way of finding a numerical solution. A few representative examples of polycondensations and non-radical polymerisations have been solved with this approach (Costa and Dias, 1994, 1995).

Numerical sensitivity already occurs with some polyadditions: it is enough to take into account the effect of the last repeating unit in the propagation or initiation rate constants in order to be struck by that problem. Parallel multiple shooting (Keller, 1972) must be used to overcome this difficulty. Free radical polymerisations present a more difficult challenge. Owing to the high relative values of termination rate constants relatively to propagation, the system becomes very "stiff" and extreme numerical sensitivity occurs.

Only recently (Costa and Dias, 2003) could a solution be found, thanks to the use of the integrator in automatic continuation code ACDC (Cash et al., 1995, 2001; Bashir-Ali et al., 1998).

In fact, its continuation procedure was not successful, as it usually leads to the trivial solution branch after gelation instead of the physical meaningful solution branch, but its implicit Runge-Kutta integrator based on a Lobatto quadrature does succeed in finding the required solutions.

Some numerical case studies will next be discussed in order to provide an overview of its expected performance in terms of accuracy, CPU time and memory requirements when dealing with complex polymerisations.

\section{Numerical treatment of non-radical polymerisation models}

It is enlightening to find the analytical solution of Flory's simplified kinetic scheme (case study I) which presents a behaviour typical of the more general chemical system; this is nearly the only way of independently checking the calculations of average molecular weights or CLD with a solution computed through a different method. Using the stoichiometric functions in Table 5, the mass balance in Laplace domain leading to the generating function of polymer NCLD in a batch reactor, neglecting density changes, may be written as follows:

$$
\begin{aligned}
\frac{\partial G}{\partial t}= & k_{I} I\left[s_{2} s_{4} s_{5} Y_{1}+2 s_{2} s_{5} s_{8} s_{9} Y_{2}\right. \\
& \left.+\frac{\partial G}{\partial \log s_{9}}\left(\frac{s_{2} s_{5}}{s_{9}}-1\right)\right] \\
& +k_{p}\left\{\frac { \partial G } { \partial \operatorname { l o g } s _ { 5 } } \left[Y_{1}\left(s_{4} s_{6}-1\right)+2 Y_{2}\left(s_{6} s_{8} s_{9}-1\right)\right.\right. \\
& \left.\left.+\frac{s_{6}}{s_{9}} \frac{\partial G}{\partial \log s_{9}}-A_{9}\right]-A_{5} \frac{\partial G}{\partial \log s_{9}}\right\} .
\end{aligned}
$$

In fact, it is possible to generalise this scheme to an arbitrary number $N_{Y}$ of monomers $Y_{j}$ with functionalities (numbers of double bonds) $j=1, N_{Y}$, provided the reactivity of all double bonds and anions is the same. This slightly more complicated scheme is discussed in Appendix B. It is convenient to introduce an overall concentration of double bonds $B$ :

$B=A_{9}+\sum_{j=1}^{N_{Y}} j Y_{j}$.

Mass balance equations of initiator, anions $A_{5}$, monomers, double bonds (adding monomer balances) and pendant double bonds $A_{9}$ can be written as:

$$
\begin{aligned}
& \frac{\mathrm{d} I}{\mathrm{~d} t}=-\frac{\mathrm{d} A_{5}}{\mathrm{~d} t}=-k_{I} I B, \\
& \frac{\mathrm{d} Y_{j}}{\mathrm{~d} t}=-j Y_{j}\left(k_{I} I+k_{p} A_{5}\right) \quad j=1, N_{Y}, \\
& \frac{\mathrm{d} B}{\mathrm{~d} t}=-B\left(k_{I} I+k_{p} A_{5}\right), \\
& \frac{\mathrm{d} A_{9}}{\mathrm{~d} t}=k_{I} I\left[-A_{9}+\sum_{j=1}^{N_{Y}} j(j-1) Y_{j}\right]-k_{p} A_{5} A_{9} .
\end{aligned}
$$

Introducing the conversions of double bonds $p$ and of initiator $p_{I}$, they verify:

$$
\begin{aligned}
& \frac{\mathrm{d} p}{\mathrm{~d} t}=I_{0}(1-p)\left[k_{I}\left(1-p_{I}\right)+k_{p} p_{I}\right], \\
& \frac{\mathrm{d} p_{I}}{\mathrm{~d} t}=k_{I} B_{0}(1-p)\left(1-p_{I}\right) .
\end{aligned}
$$


Initial weight distribution of functionalities $f_{w_{j}}$ is defined through the initial fractions of active groups belonging to each monomer $Y_{j}$ :

$f_{w_{j}}=\frac{j Y_{j_{0}}}{\sum_{j=1}^{N_{Y}} j Y_{j_{0}}}=\frac{j Y_{j_{0}}}{B_{0}}$.

The initial concentration of initiator will be given in terms of the average chain length $r$, equal to the final number average degree of polymerisation which would be achieved for $\overline{f_{w}}=1$ :

$r=\frac{B_{0}}{I_{0}}$.

Anions are associated (Lewis and Brown, 1970) in the hydrocarbon solvents used in anionic polymerisations of vinyl monomers. Rate of monomer consumption is usually not first order with respect to the overall anion concentration $A_{5}$. Although some controversy is not absent (Arest-Yakubovich, 1997; Bywater, 1998) a simple model considering association of anions $I$ and $A_{5}$ leads to a power-law dependency of apparent initiation and propagation rate constants on overall anion concentrations as shown in Eqs. (30) and (31) below, explaining observed experimental orders $\frac{1}{m_{I}}$ and $\frac{1}{m_{P}}$ (although no direct correspondence exists in many cases between degree of aggregation and these parameters, as shown by Young et al. (1984))

$k_{I}=k_{I_{0}} I^{-1+1 / m_{I}}$,

$k_{p}=k_{p_{0}} I^{-1+1 / m_{p}}$.

An example is $n$-butyllithium initiated bulk polymerisation of styrene in benzene, for which $m_{I}=6$ (Worsfold and Bywater, 1960), and $m_{P}=2$.

Karles et al. (1991) have found that $m_{P}=1.2 \pm 0.8$ for bulk polymerisation of DVB, different from styrene, which is a source of some complications in kinetic modelling. Notice that no mixed association of anions coming from initiator and from polymer would be allowed using this simplified model (which does not allow either for dependence of $m_{P}$ on the nature of terminal unit). According to Eqs. (30), (31) and (27), the two conversions would be related through a binomial integral:

$p=\frac{k_{I_{0}}}{k_{p_{0}}} I_{0}^{\frac{1}{m_{I}}-\frac{1}{m_{p}}} \int_{0}^{p_{I}} u^{1-\frac{1}{m_{p}}}(1-u)^{\frac{1}{m_{I}}-1} \mathrm{~d} u$.

Owing to the binomial integral in Eq. (32) above, a full analytical solution is possible only for instantaneous initiation or for a constant ratio of initiation and propagation apparent constants $C_{I}=\frac{k_{I}}{k_{p}}$. If this condition holds, conversions of initiator $p_{I}$ and of active groups in monomers $p$ are related through:

$\log \left(1-p_{I}\right)=\left(C_{I}-1\right) p_{I}-r C_{I} p$.
The critical conversion for gelation $p_{g}$, related to $p_{I_{g}}$ through Eq. (33) above, results from the calculations in Appendix B:

$$
\begin{aligned}
\frac{1}{\overline{f_{w}}-1}= & r p_{g}^{2}-\left(1-1 / C_{I}\right)\left\{2\left(1-1 / C_{I}\right) p_{g}\right. \\
& \left.+\left[\left(1-1 / C_{I}\right) p_{I_{g}}-2\right] p_{I_{g}} / r\right\} .
\end{aligned}
$$

For a given initial weight average monomer functionality, gelation occurs only if the initial mole ratio $r$ is above a critical value $r_{c}$ obtained by setting $p_{g}=p_{I_{g}}=1$ in Eq. (34) above.

As previously stated, inclusion of hydride expulsion and transfer to solvent, which are important reactions unless temperature is much lower than ambient (Priddy et al., 1992), makes impossible to find an analytical solution for the prediction of average molecular weights.

The set of kinetic parameters and initial concentrations used in case studies I and II is presented in Table 8. Values for relative reactivities of double bonds were taken from Worsfold (1970) and rate constants for secondary reactions were taken from Priddy et al. (1992). Some values had to be assumed owing to lack of experimental data.

We have considered three variants of the kinetic scheme:

- System I: as described in Table 8;

- System II: equal reactivities of double bonds and anions, as would approximately hold for styrene $+m$ divinylbenzene;

- System III: as system II without transfer to solvent and termination, corresponding to case study I.

The analytical solution (case study I, system III) was precious in order to test the numerical solution method. A very serious problem, as shown in Fig. 1, is the numerical sensitivity of system of characteristics Eqs. (A.1)-(A.6), forcing the use of parallel multiple shooting (Keller, 1972), as we have already done in an earlier research (Costa and Dias, 1995) with a "home-made" code.

Notice in Fig. 2 the very different shapes of characteristic curves associated to the different variables; characteristics associated to the numbers of anions are smooth (Fig. 3), while those associated to the numbers of double bonds have a steep growth towards the end of the integration interval (Fig. 4).

Code ACDC (Cash et al., 2001) can deal with the "stiff" systems (Hairer and Wanner, 2002) found in free-radical polymerisation (discussed in next section) and this is a relatively easy problem for that method. Prediction of weight-average molecular weight takes about $30 \mathrm{~s} \mathrm{CPU}$ before gelation, and each prediction of sol fraction and $\bar{M}_{w}$ after gelation takes about $59 \mathrm{~s}$. Computations were performed with a PIV $1.5 \mathrm{GHz}$ running Linux (computing times are about the same with a dual G4 Power PC running Apple MacOSX, also with a program compiled with GNU C compiler gcc-2.95 and associated Fortran compiler). 
Table 8

Kinetic parameters for simulations of anionic copolymerisation of styrene $+p$-divinylbenzene (case study II)

\begin{tabular}{|c|c|c|}
\hline Parameter & Relative value & Absolute value \\
\hline$M_{1_{0}}$ & & $3.996 \mathrm{~mol} \mathrm{dm}^{-3}$ \\
\hline$M_{2_{0}}$ & & $0.004 \mathrm{~mol} \mathrm{dm}^{-3}$ \\
\hline$I_{0}$ & & $0.004 \mathrm{~mol} \mathrm{dm}^{-3}$ \\
\hline$S_{0}$ & & $4 \mathrm{~mol} \mathrm{dm}^{-3}$ \\
\hline$k_{I}$ & & $0.7 \mathrm{dm}^{3} \mathrm{~mol}^{-1} \mathrm{~s}^{-1}$ \\
\hline$k_{p}$ & & $220 \mathrm{dm}^{3} \mathrm{~mol}^{-1} \mathrm{~s}^{-1}$ \\
\hline$k_{S}$ & & $1.4 \times 10^{-4} \mathrm{dm}^{3} \mathrm{~mol}^{-1} \mathrm{~s}^{-1}$ \\
\hline$k_{e}$ & & $2 \times 10^{-4} s^{-1}$ \\
\hline$k_{p_{11}}$ & $k_{p_{11}}=k_{p}$ & \\
\hline$k_{p_{22}}$ & $C_{p}=k_{p_{22}} / k_{p_{11}}=9.7$ & \\
\hline$k_{p_{12}}$ & $r_{1}=k_{p_{11}} / k_{p_{12}}=0.094$ & \\
\hline$k_{p_{21}}$ & $r_{2}=k_{p_{22}} / k_{p_{21}}=10$ & \\
\hline$k_{p_{13}}$ & $C_{p_{1}}=k_{p_{13}} / k_{p_{11}}=0.8$ & \\
\hline$k_{p_{23}}$ & $C_{p_{2}}=k_{p_{23}} / k_{p_{22}}=0.07$ & \\
\hline$k_{I_{1}}$ & $C_{I_{1}}=k_{I_{1}} / k_{p_{11}}=k_{I} / k_{p}=0.0032$ & \\
\hline$k_{I_{2}}$ & $C_{I_{2}}=k_{I_{2}} / k_{p_{12}}=C_{I_{1}}=0.0032$ & \\
\hline$k_{I_{3}}$ & $C_{I_{3}}=k_{I_{3}} / k_{p_{13}}=C_{I_{1}}=0.0032$ & \\
\hline$k_{I_{4}}$ & $C_{I_{4}}=k_{I_{4}} / k_{p_{11}}=C_{I_{1}}=0.0032$ & \\
\hline$k_{I_{5}}$ & $C_{I_{5}}=k_{I_{5}} / k_{p_{12}}=C_{I_{1}}=0.0032$ & \\
\hline$k_{I_{6}}$ & $C_{I_{6}}=k_{I_{6}} / k_{p_{13}}=C_{I_{1}}=0.0032$ & \\
\hline$k_{e_{1}}$ & $k_{e_{1}}=k_{e}$ & \\
\hline$k_{e_{2}}$ & $k_{e_{2}}=k_{e}$ & \\
\hline$k_{t_{11}}$ & $k_{t_{11}}=k_{p_{11}}$ & \\
\hline$k_{t_{12}}$ & $k_{t_{12}}=k_{p_{12}} / 2$ & \\
\hline$k_{t_{21}}$ & $k_{t_{21}}=k_{p_{12}}$ & \\
\hline$k_{t_{22}}$ & $k_{t_{22}}=k_{p_{22}} / 2$ & \\
\hline$k_{S_{1}}$ & $k_{S_{1}}=k_{S}$ & \\
\hline$k_{S_{2}}$ & $k_{S_{2}}=k_{S}$ & \\
\hline
\end{tabular}

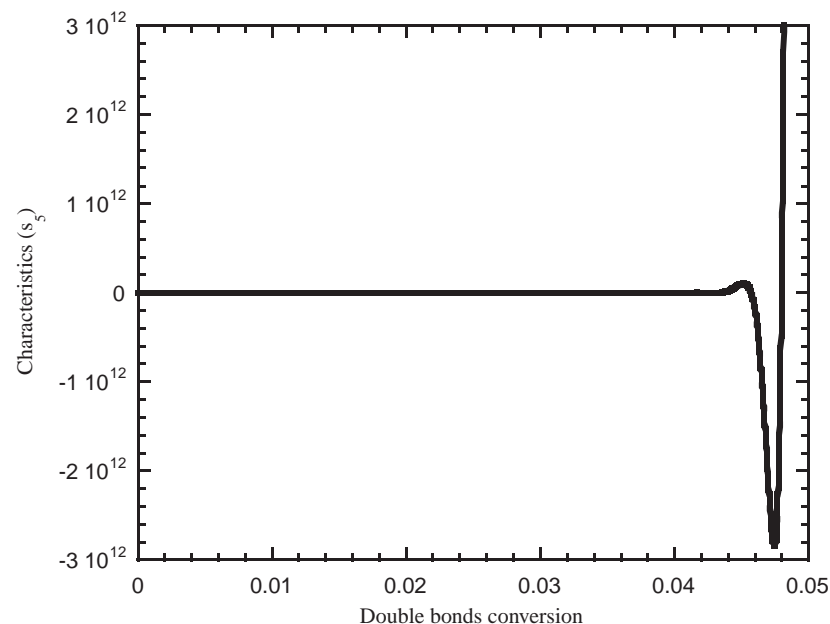

Fig. 1. Numerical "blow-up" of solution of system of differential equations for computing characteristics.

Numerical accuracy can be checked for system III comparing predicted values of $G$ obtained with the analytical solution described in Appendix B. In fact, this solution involves the numerical resolution of the algebraic equation Eq. (B.12) or Eq. (B.13), which is rather tricky for high dou-

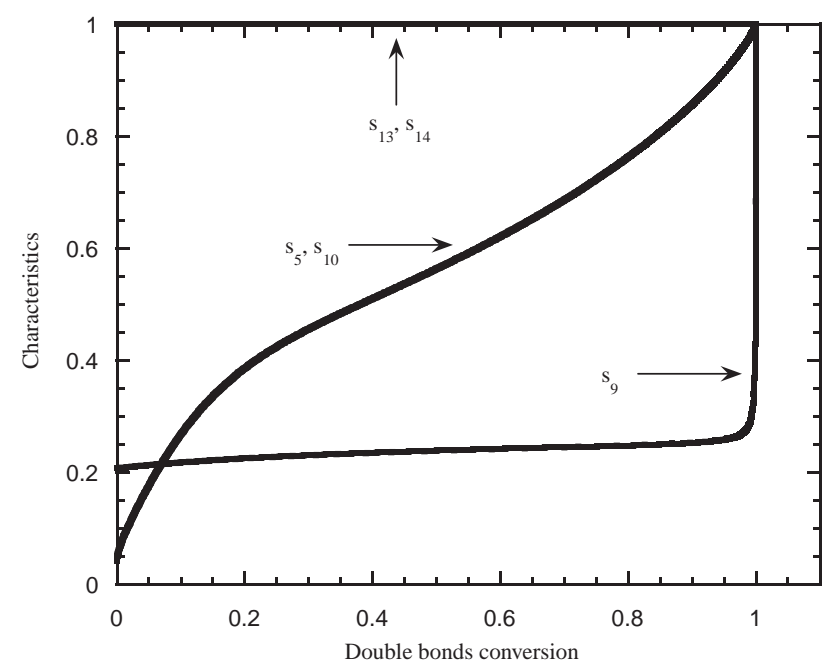

Fig. 2. Characteristics for case study II, system I, at $p=1$.

ble bond conversions and so it can have a non-negligible numerical error too.

Nevertheless, in the worst case (conversion $p=0.999$ ), there is agreement of $G(\mathbf{1})$ by the two methods within 9 decimal places, and except for that extreme case we have 


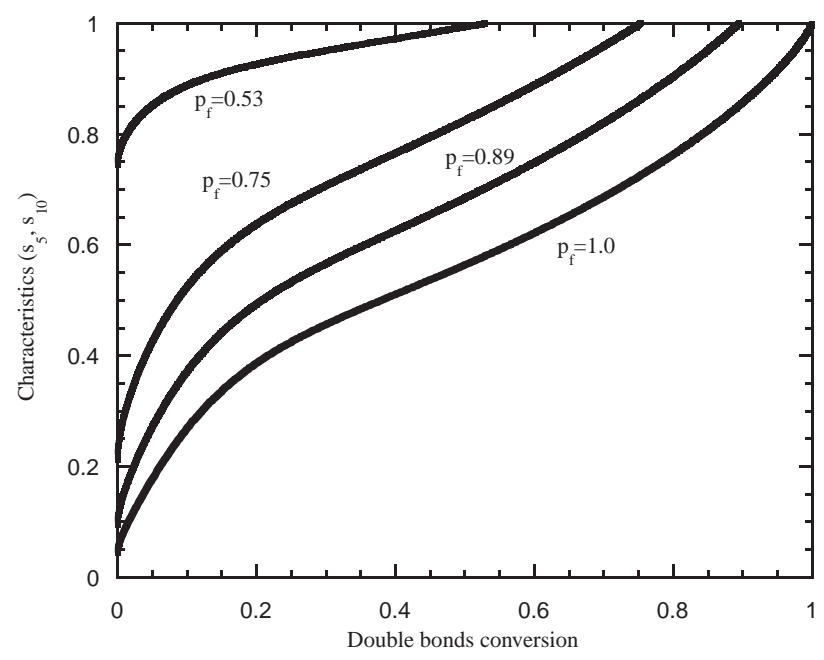

Fig. 3. Characteristics for case study II, system I, for different values of double bonds conversion $p$.

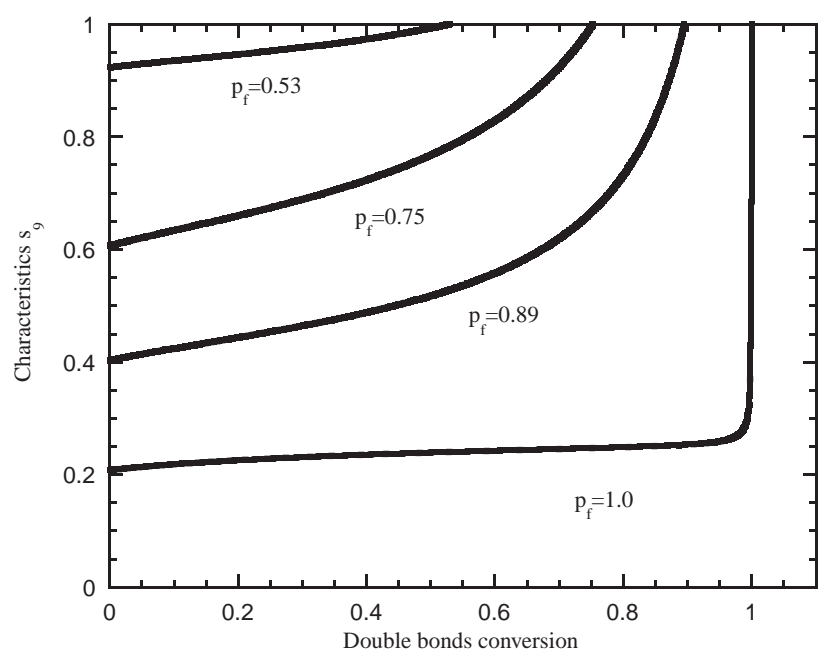

Fig. 4. Characteristics for case study II, system I, for different values of double bonds conversion $p$.

obtained 11 decimal places agreement, so this should be regarded as satisfactory.

In Fig. 5 is shown an example of number and weight average molecular weights prediction, while in Figs. 6 and 7 are compared the changes brought up by the different values of rate parameters.

Unfortunately, no experimental data for molecular weight have been published for these systems.

\section{Modelling of free radical copolymerisation of monovinyl and divinyl monomers and its application to styrene + divinylbenzene systems (case study III)}

In order to present a comparison of predictions of the present method with experimental data, the classical prob-

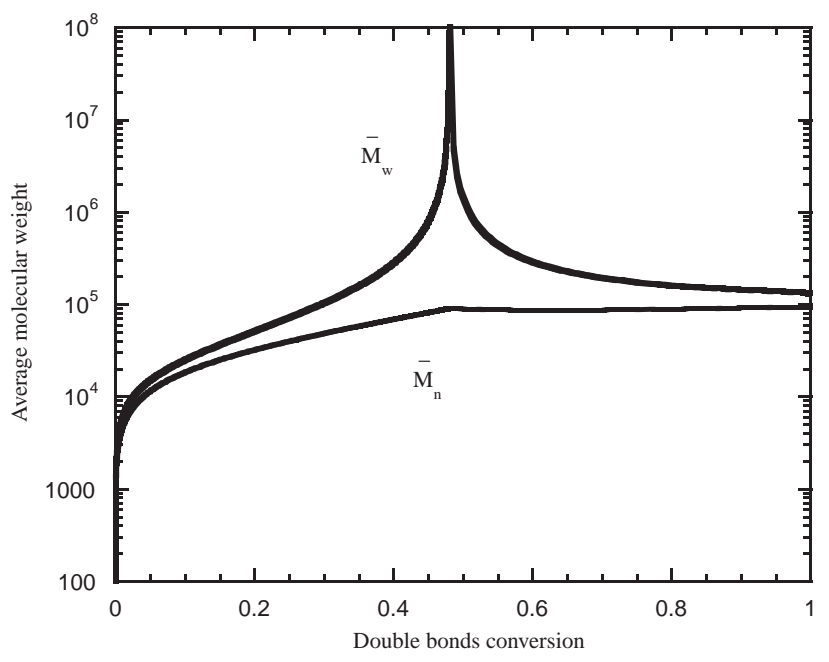

Fig. 5. $\bar{M}_{n}$ and $\bar{M}_{w}$ for case study II.

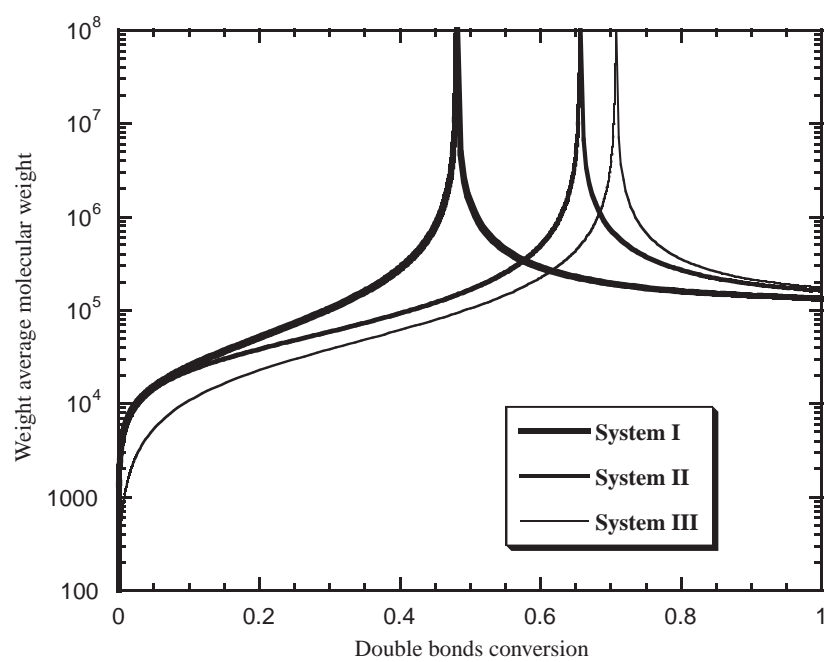

Fig. 6. $\bar{M}_{w}$ for case study II with different kinetics.

lem of the free radical crosslinking copolymerisation will be used as an example. For this kind of systems, some experimental studies can be found in the literature namely for the copolymerisation of mono/divinyl monomers. It was important for our goals to have available experimental data in the pre- and post-gel regimes, and the extensive investigation on kinetics of free radical copolymerisation, in benzene and $60^{\circ} \mathrm{C}$, of styrene with $m$ - and $p$-divinylbenzene, ethylene dimethacrylate and diisopropenylbenzene (Hild and Okasha, 1985a,b; Hild et al., 1985) could fulfil these requirements. This chemical system should only present a low extent of cyclisations, which are an important factor in most nonlinear free-radical polymerisations (see Elliott and Bowman (2002) as an example of a sustained modelling effort of these systems).

Variable, but always small, mole ratios of divinyl monomer to styrene have been used in these experiments. 


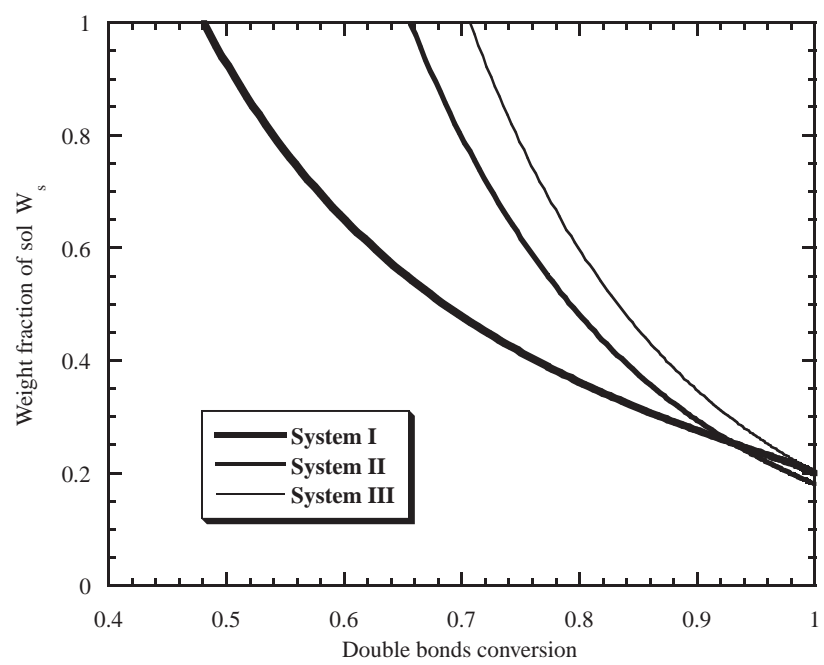

Fig. 7. Weight fraction of sol for case study II with different kinetics.

Table 9

Description of groups considered in the free radical crosslinking copolymerisation of styrene with $m$-divinylbenzene

\begin{tabular}{llll}
\hline Group description & $j$ & $\delta_{P_{j}}$ & $\delta_{A_{j}}$ \\
\hline Free radical from styrene & 1 & 1 & 1 \\
Free radical from $m$-divinylbenzene & 2 & 1 & 1 \\
Pendant double bond from $m$-divinylbenzene & 3 & 1 & 1 \\
Monomeric styrene & 4 & 0 & 1 \\
Monomeric $m$-divinylbenzene & 5 & 0 & 1 \\
Primary radical & 6 & 0 & 1 \\
Initiator (AIBN) & 7 & 0 & 1 \\
& & & \\
Polymerised styrene unit & 8 & 1 & 0 \\
Polymerised $m$-divinylbenzene unit & 9 & 1 & 0 \\
\hline
\end{tabular}

System styrene/m-divinylbenzene was investigated before gelation with initial concentrations $M_{1_{0}}=4 \mathrm{~mol} \mathrm{dm}^{-3}$ for styrene, $M_{2_{0}}=0.08 \mathrm{~mol} \mathrm{dm}^{-3}$ for $m$-divinylbenzene and $I_{0}=0.08 \mathrm{~mol} \mathrm{dm}^{-3}$ for AIBN. This corresponds to an initial mole ratio of divinyl monomer with respect to monovinyl monomer of $\alpha=\frac{M_{2_{0}}}{M_{1}}=0.02$ (2\%). The same system was studied after gelation for an initial composition of $M_{1_{0}}=4 \mathrm{~mol} \mathrm{dm}^{-3}, M_{2_{0}}=0.2 \mathrm{~mol} \mathrm{dm}^{-3}$ and $I_{0}=0.08 \mathrm{~mol} \mathrm{dm}^{-3}$ which corresponds to $\alpha=5 \%$. Average molecular weights have been measured by size exclusion chromatography (SEC) and in some cases by light scattering (LS) and are available in these works in a tabular form.

A description of groups and kinetic scheme for the simulation of this system is presented in Tables 9 and 10. Free radicals from styrene, free radicals from $m$-divinylbenzene and pendant double bonds from $m$-divinylbenzene are the active species present in the polymer. Monomers, primary radicals and initiator are active but are not present in the polymer. Polymerised monomer units are present in the polymer but are inactive. The kinetic scheme comprises the usual steps in this kind of systems: initiator decomposition, initiation of monomers and pendant double bonds with primary radicals, propagation of monomers and pendant double bonds with the different kinds of radicals and termination between the different polymeric radicals, which in this systems is known to occur predominantly by combination.

Kinetic parameters assumed in this case study are presented in Table 11. A set of parameters were fixed as reference values (Hild and Okasha, 1985a): $k_{p 11}$ is the rate constant of homopropagation of styrene and $k_{t c}$ is the correspondent value termination rate constant, $f=0.6$ was kept fixed and $k_{d}$ a known value for the dissociation of AIBN.

Recent experimental studies (Zetterlund et al., 2002) have found that apparent termination rate constant and initiator efficiency are neither independent from conversion nor from divinylbenzene content. We have nevertheless kept using earlier found values because they fit these observed conversion vs. time values.

Reactivity ratio of styrene $r_{1}$ was experimentally determined in that work, where it is also reported the impossibility of a precise determination of the reactivity ratio for $m$-divinylbenzene due to the small amount of this monomer in the initial reaction mixture. In these conditions, an ideal copolymerisation has been assumed: $r_{1} r_{2}=1$. Only with a strong deviation from ideality a noticeable influence of this parameter in the gelation of the system (Costa and Dias, 2003) is expected. Conversely, reactivity of pendant double bonds has a strong influence in the behaviour of this kind of systems. Its relative value to the propagation of styrene $\left(C_{p_{13}}\right)$ is used as a reference value. The remaining kinetic parameters needed for the simulations can be obtained from this basic set as reported in Table 11. Here the present simulation method was used to obtain an estimation of $C_{p_{13}}$ : the observed gelation time for the experimental system with $\alpha=2 \%, t_{g}=7.5 \mathrm{~h}$ could be reproduced and $C_{p_{13}}=0.138$ was estimated with a relative error of $0.06 \%$ in the gelation time.

For these reaction conditions, in Fig. 8 are compared the predictions of the present method with experimental values for weight and number average molecular weights (Hild and Okasha, 1985a) in the polymers produced with $\alpha=2 \%$. Some discrepancies were observed between the experimentally measured weight average molecular weight by SEC and LS, namely in the neighbourhood of gelation. Nevertheless, the agreement between predictions and experimental values is fairly good.

With the same set of kinetic parameters, the system with $\alpha=5 \%$ was also simulated and a comparison with the correspondent experimental values obtained by Hild et al. (1985) can be found in Fig. 9; in this case (post-gel results) only experimental observations by SEC are available. At first sight, agreement is not bad either, but, unfortunately, inconsistent results are found: predicted gelation time is $t_{g}=2.1 \mathrm{~h}$ while the experimental value is $t_{g}=3.5 \mathrm{~h}$. In order to reproduce the reported gelation time, a new value of $C_{p_{13}}=0.091$ was 
Table 10

Reaction scheme in the free radical crosslinking copolymerisation of styrene with $m$-divinylbenzene

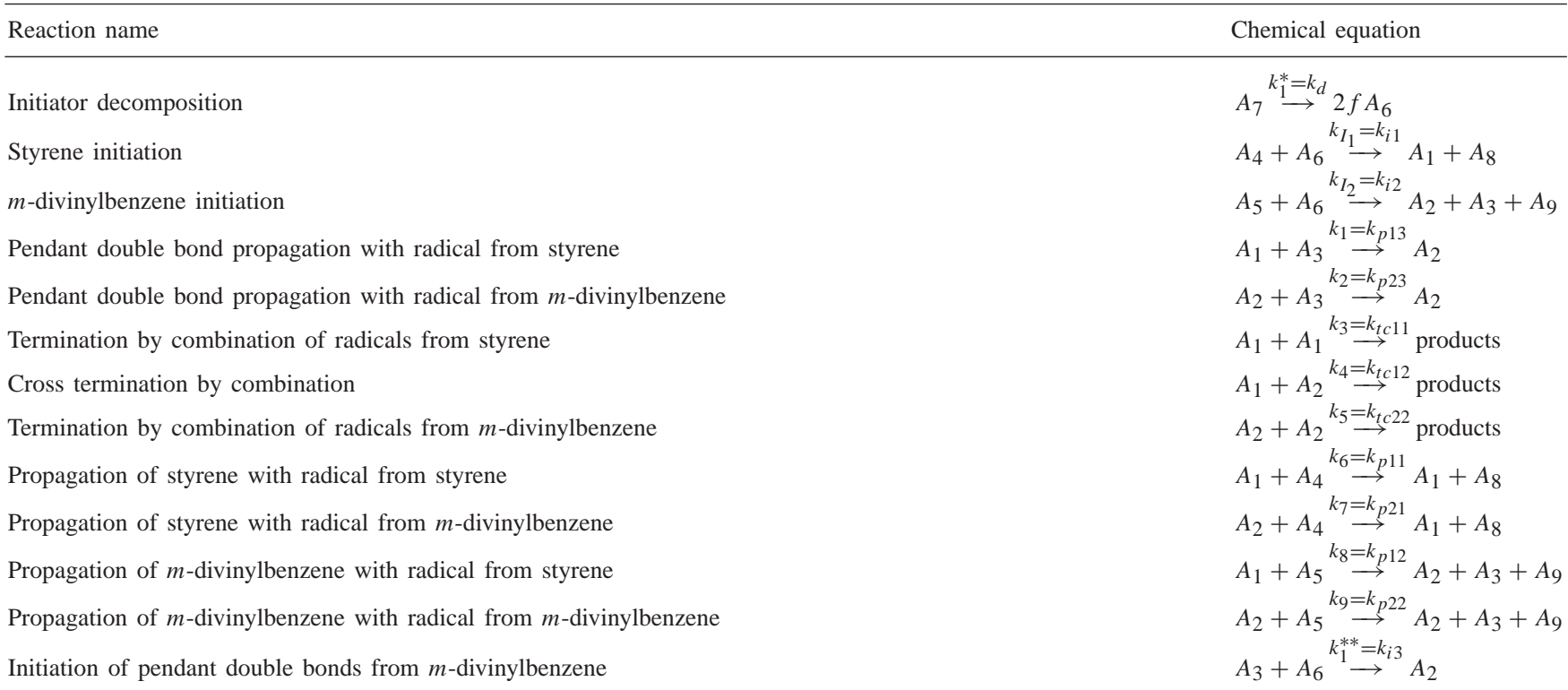

Table 11

Kinetic parameters in free radical crosslinking copolymerisation of styrene with $m$-divinylbenzene

\begin{tabular}{|c|c|c|}
\hline Kinetic constant & Relative value & Absolute value \\
\hline$k_{p 11}$ & & $145 \mathrm{dm}^{3} \mathrm{~mol}^{-1} \mathrm{~s}^{-1}$ \\
\hline$k_{t c}$ & & $2.9 \times 10^{7} \mathrm{dm}^{3} \mathrm{~mol}^{-1} \mathrm{~s}^{-1}$ \\
\hline$k_{d}$ & & $8.5 \times 10^{-6} \mathrm{~s}^{-1}$ \\
\hline$f$ & & 0.6 \\
\hline$r_{1}$ & $r_{1}=\frac{k_{p 11}}{k_{p 12}}$ & 0.44 \\
\hline$k_{p 13}$ & $C_{p_{13}}=\frac{k_{p 13}}{k_{p 11}}=0.138$ & $20.01 \mathrm{dm}^{3} \mathrm{~mol}^{-1} \mathrm{~s}^{-1}$ \\
\hline$k_{i 1}$ & $C_{i 1}=\frac{k_{i 1}}{k_{p 11}}=1$ & $145 \mathrm{dm}^{3} \mathrm{~mol}^{-1} \mathrm{~s}^{-1}$ \\
\hline$k_{i 2}$ & $C_{i 2}=\frac{k_{i 2}}{k_{p 11}}=\frac{1}{r_{1}}$ & $329.5 \mathrm{dm}^{3} \mathrm{~mol}^{-1} \mathrm{~s}^{-1}$ \\
\hline$k_{i 3}$ & $C_{i 3}=\frac{k_{i 3}}{k_{p 11}}=C_{p_{13}}$ & $20.01 \mathrm{dm}^{3} \mathrm{~mol}^{-1} \mathrm{~s}^{-1}$ \\
\hline$k_{p 12}$ & $r_{1}=\frac{k_{p 11}}{k_{p 12}}$ & $329.5 \mathrm{dm}^{3} \mathrm{~mol}^{-1} \mathrm{~s}^{-1}$ \\
\hline$k_{p 21}$ & $C_{p_{21}}=\frac{k_{p 21}}{k_{p 11}}=1$ & $145 \mathrm{dm}^{3} \mathrm{~mol}^{-1} \mathrm{~s}^{-1}$ \\
\hline$k_{p 22}$ & $r_{2}=\frac{k_{p 22}}{k_{p 21}}=\frac{1}{r_{1}}$ & $329.5 \mathrm{dm}^{3} \mathrm{~mol}^{-1} \mathrm{~s}^{-1}$ \\
\hline$k_{p 23}$ & $C_{p_{23}}=\frac{k_{p 23}}{k_{p 11}}=C_{p_{13}}$ & $20.01 \mathrm{dm}^{3} \mathrm{~mol}^{-1} \mathrm{~s}^{-1}$ \\
\hline$k_{t c 11}$ & & $2.9 \times 10^{7} \mathrm{dm}^{3} \mathrm{~mol}^{-1} \mathrm{~s}^{-1}$ \\
\hline$k_{t c 12}$ & & $2.9 \times 10^{7} \mathrm{dm}^{3} \mathrm{~mol}^{-1} \mathrm{~s}^{-1}$ \\
\hline$k_{t c 22}$ & & $2.9 \times 10^{7} \mathrm{dm}^{3} \mathrm{~mol}^{-1} \mathrm{~s}^{-1}$ \\
\hline
\end{tabular}

calculated and the correspondent simulations have also been compared with experimental results in Fig. 9. Agreement is not so good as the observed with the previous set of kinetic parameters.

A similar apparent reactivity decrease of pendant double bonds with increasing amounts of divinyl monomer has been reported with this same chemical system (Zetterlund et al., 2002) as well as many others, namely by Tobita and Hamielec (1989) in their analysis of the copolymerisation of methyl methacrylate with ethylene glycol dimethacrylate. The effect of cyclisation has been pointed as a contributing factor for this kind of inconsistencies observed in these chemical systems, but this is unlikely for this system. 


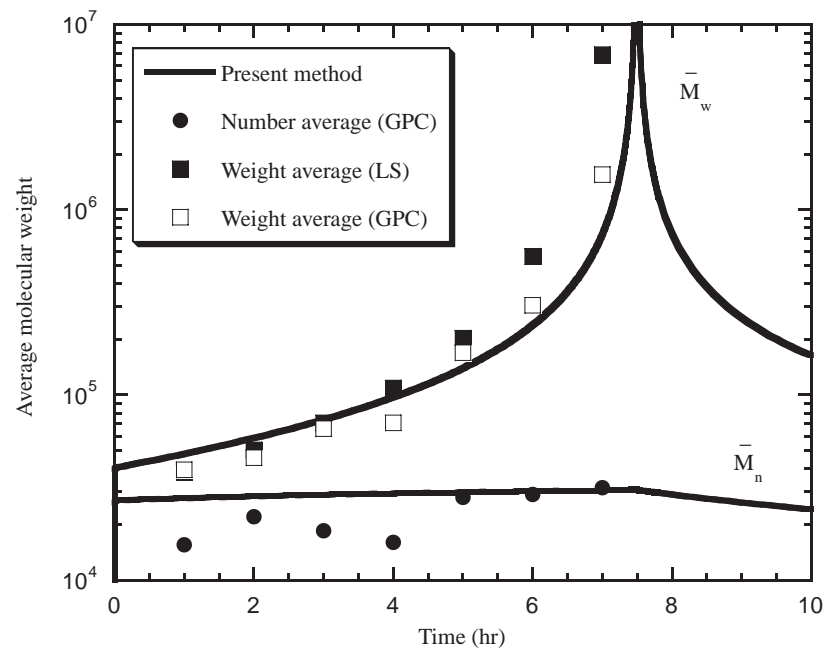

Fig. 8. Comparison between the predictions of the present method and experimental data (Hild and Okasha, 1985a) for the free radical crosslinking copolymerisation of styrene and $m$-divinylbenzene with an initial mole ratio $\alpha=2 \%$.

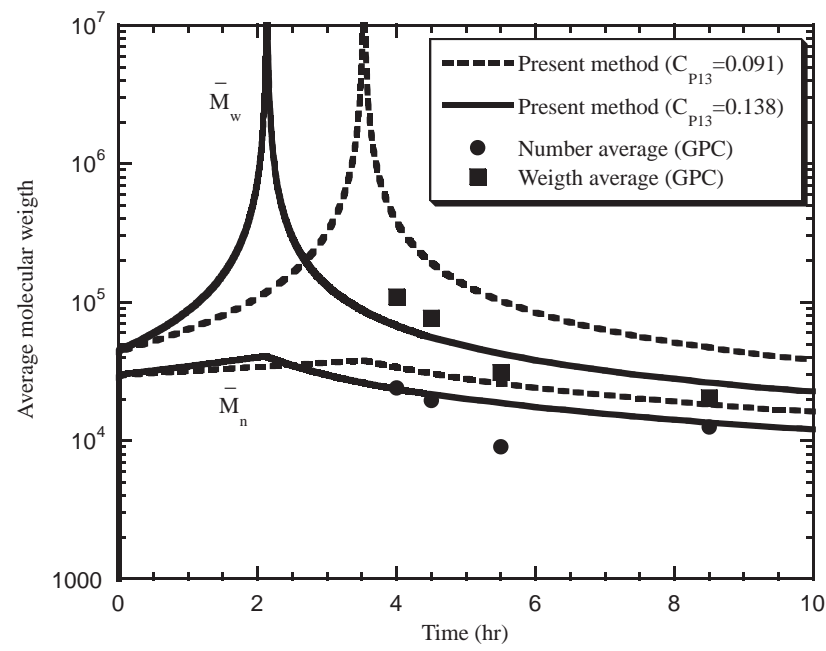

Fig. 9. Comparison between the predictions of the present method and experimental data (Hild et al., 1985) for the free radical crosslinking copolymerisation of styrene and $m$-divinylbenzene with an initial mole ratio $\alpha=5 \%$.

In our opinion, the problem should rather involve the modelling of the termination reaction and the assumed constant initiator efficiency. Further investigation is needed in this subject, namely to clarify the adequacy of the kinetic schemes involved in these kind of polymerisations and to obtain more reliable experimental results, particularly in the post-gel region.

Owing to its improved mathematical exactness, the ability to deal with gel and the possibility to consider complex kinetic schemes, the method presented in this work could be a valuable tool for this purpose.

\section{Modelling of free radical polymerisation with transfer to polymer (case study IV)—a comparison with other approaches}

As free-radical polymerisations are kinetically controlled, the condition for random branch points in a statistical gelation process often cannot be used. This fact invalidates the mathematical treatment of this systems by the theory of branching processes.

Modelling non-linear free radical polymerisations has been for a long time a controversial subject. Based on pioneering work by Bamford and Tompa (1954), several models have been applied to the treatment of non-linear free radical polymerisations. These models have been derived by writing population balances in the reactor and a set of ordinary differential equations results for the moments of the CLD (method of the moments). Nevertheless, in order to obtain a tractable model, some mathematical approximations have been introduced, such as (among others) pseudo-steady state for radical concentrations and negligible presence of multiple radical centres. Additionally, it is necessary to consider closure conditions to make possible the resolution of the equations for the moments. Extension of the method of the moments to the post gel region remains difficult (Tobita and Hamielec, 1989). Therefore the method of moments or related methods that use this kind of population balances are mostly used to the analysis of pregel conditions and a set of approximation conditions has to be used (Miller et al., 1996; Asteasuain et al., 2002a,b).

Important discrepancies can be observed when predictions by the method of moments are compared with more accurate methods (Costa and Dias, 2003).

Monte Carlo simulation has the advantage of providing a detailed view of molecular structure and is a possible alternative method for dealing with these problems. Its successful use with polycondensations has been known since long ago (Mikeš and Dušek, 1982). However, widely different time scales in free radical polymerisation make its straightforward application very time consuming, and so some simplifications had to be introduced (Tobita, 1994b,c).

Recent simulations of non-linear free radical polymerisations using Galerkin finite-elements method (Iedema and Hoefsloot, 2002) have once again neglected the presence of multiple radical centres together with some simplifications in the kinetic schemes. In addition, the properties of the gel cannot be obtained by this method.

"Numerical fractionation" (Teymour and Campbell, 1994) overcomes many difficulties associated with the use of the method of moments when gel is present and can be used for reconstructing an approximate CLD. Nevertheless, this technique is based on population balances making use of many approximations also present in the method of moments.

For comparison purposes of theoretical predictions, the free radical polymerisation of a single monomer with transfer to polymer in a batch reactor has been chosen. This is a 
Table 12

Description of groups in a free radical polymerisation with transfer to polymer

\begin{tabular}{|c|c|c|c|c|}
\hline Group description & $j$ & $\delta_{P_{j}}$ & $\delta_{A_{j}}$ & Chemical formula (example) \\
\hline Free radical & 1 & 1 & 1 & $-\mathrm{HYC}^{\bullet}$ \\
\hline Transfer to polymer center & 2 & 1 & 1 & $-\mathrm{CH}_{2}-\mathrm{CHY}-\mathrm{CH}_{2}-$ \\
\hline Monomer & 3 & 0 & 1 & $\mathrm{CH}_{2}=\overline{\mathrm{CHY}}$ \\
\hline Primary radical & 4 & 0 & 1 & $\left(\mathrm{CH}_{3}\right)_{2} \mathrm{CNC}^{\bullet}$ \\
\hline Initiator & 5 & 0 & 1 & $\left(\mathrm{CH}_{3}\right)_{2} \mathrm{CNCN}=\mathrm{NCCN}\left(\mathrm{CH}_{3}\right)_{2}$ \\
\hline Polymerised monomer unit & 6 & 1 & 0 & $-\mathrm{CH}_{2}-\mathrm{CHY}-$ \\
\hline
\end{tabular}

Table 13

Reaction scheme in a free radical polymerisation with transfer to polymer

\begin{tabular}{ll}
\hline Reaction name & Chemical equation \\
\hline Initiator decomposition & $A_{5} \stackrel{k_{1}^{*}=k_{d}}{\longrightarrow} 2 f A_{4}$ \\
Monomer initiation & $A_{3}+A_{4} \stackrel{k_{1}=k_{i}}{\longrightarrow} A_{1}+A_{6}$ \\
Termination by combination & $A_{1}+A_{1} \stackrel{k_{1}=k_{t c}}{\longrightarrow}$ products \\
Propagation of monomer & $A_{1}+A_{3} \stackrel{k_{2}=k_{p}}{\longrightarrow} A_{2}+A_{1}+A_{6}$ \\
Transfer to polymer & $A_{1}+A_{2} \stackrel{k_{1}^{* *}=k_{f p}}{\longrightarrow} A_{1}$ \\
Termination by disproportionation & $A_{1}+A_{1} \stackrel{k_{2}^{* *}=k_{t d}}{\longrightarrow}$ products \\
\hline
\end{tabular}

classical problem which has been analysed in the past by the method of the moments (Tobita and Hamielec, 1988) and "numerical fractionation" (Teymour and Campbell, 1994). The description of groups and kinetic scheme considered is presented in Tables 12 and 13. Kinetic parameters for this case study are presented in Table 14 . The propagation rate constant for the monomer is a recently measured value by PLP for vinyl acetate (Hutchinson et al., 1994). Initiator decomposition rate constant and efficiency were taken as usual values for AIBN.

Initial monomer and initiator concentration has been kept fixed at, respectively, $M_{0}=3 \mathrm{moldm}^{-3}$ and $I_{0}=$ $4.29 \times 10^{-3} \mathrm{~mol} \mathrm{dm}^{-3}$. Remaining parameters in Table 14 were chosen in order to specify simulation conditions close to the used in other analysis of this polymerisation system (Teymour and Campbell, 1994; Tobita and Hamielec, 1988).

For these reaction conditions, predictions of "numerical fractionation" technique have been obtained according to the principles presented in literature (Teymour and Campbell, 1994). Saidel-Katz approximation for the third moment was used as closure condition and $n=10$ generations were considered in the calculations.

In Fig. 10 are presented the predictions by the two methods for the number- and weight-average chain lengths as a function of monomer conversion. Number-average chain length shows only small differences in the post gel region. Nevertheless, for the weight average chain length the predictions present important differences in the vicinity of the gel point and beyond. To test the consistency of the methods the linear case $\left(C_{P}=0\right)$ has also been simulated, and, as expected, no significant differences have been observed (Fig. 11).

This fact, in our opinion, is explained by the effect of the approximation conditions used in "numerical fractionation", namely the neglect of the existence of poly-radicals, which are known to exist in fairly large amounts close to gelation (Tobita and Zhu, 1996).

\section{Conclusions}

This work could offer some significant steps towards building a kind of interpreter of irreversible polymerisation schemes. Fairly general and complex chemical systems can be analysed without the need for approximations like pseudo-steady state hypothesis. Any number of radical or ion sites can exist in a single molecule, and this helps to eliminate approximations or even inconsistencies that arise from treating non-linear polymerisations as a perturbation of linear polymerisation schemes (Costa and Dias, 2003).

Intrinsic mathematical limitations of commercial software dealing with polymerisation reactions and reactors could therefore be overcome.

Practical implementation of this approach shows that computational resources needed for prediction of average molecular weights before gelation are quite modest for current personal computers.

Prediction of sol fraction and average molecular weights after gelation is a much more difficult problem, especially for free radical polymerisation, and some tens of hours of CPU time and 512 or more of RAM with a personal computer are the bare minimum.

Predictions of average molecular weights and gelation time for styrene $+m$-divinylbenzene assuming no intramolecular reaction could not fit experimental data using a single value of relative reactivity of pending double bonds for different initial mole ratios of divinyl monomer. Even if this chemical system should not be too sensitive to intramolecular reaction, this factor is possibly at the origin of these discrepancies. It is expected that the computational approach described in this paper can help in further investigations of these polymerisations, as it can be readily extended to encompass other chemical reactions, such as intramolecular propagation, transfer and termination. 
Table 14

Kinetic parameters in free radical polymerisation with transfer to polymer

\begin{tabular}{|c|c|c|}
\hline Kinetic constant & Relative value & Absolute value \\
\hline$k_{p}$ & & $1.17 \times 10^{4} \mathrm{dm}^{3} \mathrm{~mol}^{-1} \mathrm{~s}^{-1}$ \\
\hline$k_{d}$ & & $9 \times 10^{-6} s^{-1}$ \\
\hline$f$ & & 0.5 \\
\hline$k_{i}$ & $C_{i}=\frac{k_{i}}{k_{p}}=1.0$ & $1.17 \times 10^{4} \mathrm{dm}^{3} \mathrm{~mol}^{-1} \mathrm{~s}^{-1}$ \\
\hline$k_{f p}$ & $C_{P}=\frac{k_{f p}}{k_{p}}=10^{-3}$ & $11.7 \mathrm{dm}^{3} \mathrm{~mol}^{-1} \mathrm{~s}^{-1}$ \\
\hline$k_{t d}$ & & $17.55 \times 10^{7} \mathrm{dm}^{3} \mathrm{~mol}^{-1} \mathrm{~s}^{-1}$ \\
\hline$k_{t c}$ & $C_{t c}=\frac{k_{t c}}{k_{t d}}=0.1$ & $1.755 \times 10^{7} \mathrm{dm}^{3} \mathrm{~mol}^{-1} \mathrm{~s}^{-1}$ \\
\hline
\end{tabular}

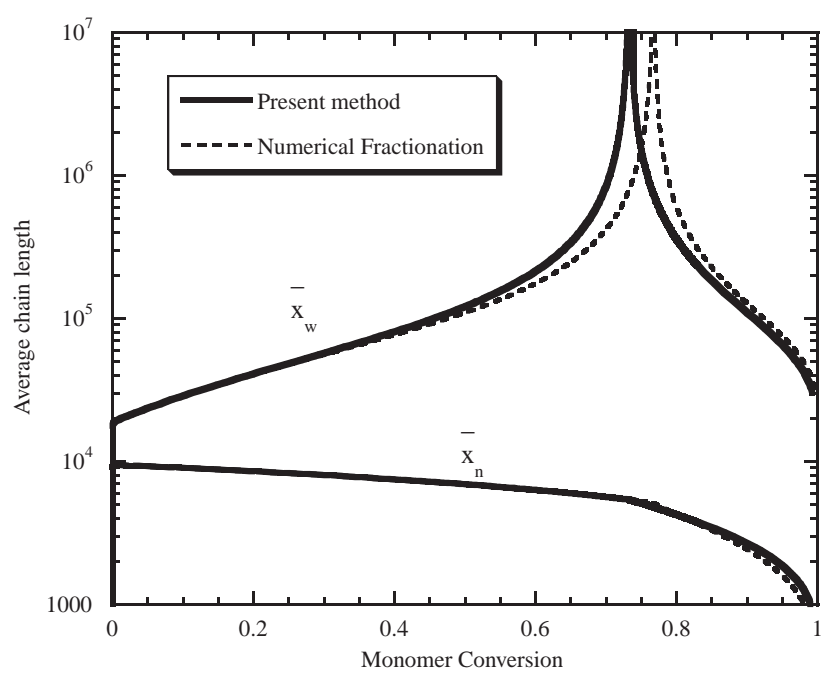

Fig. 10. Comparison between the predictions of the present method and "Numerical Fractionation" technique for a non-linear free radical polymerisation with transfer to polymer in a batch reactor.

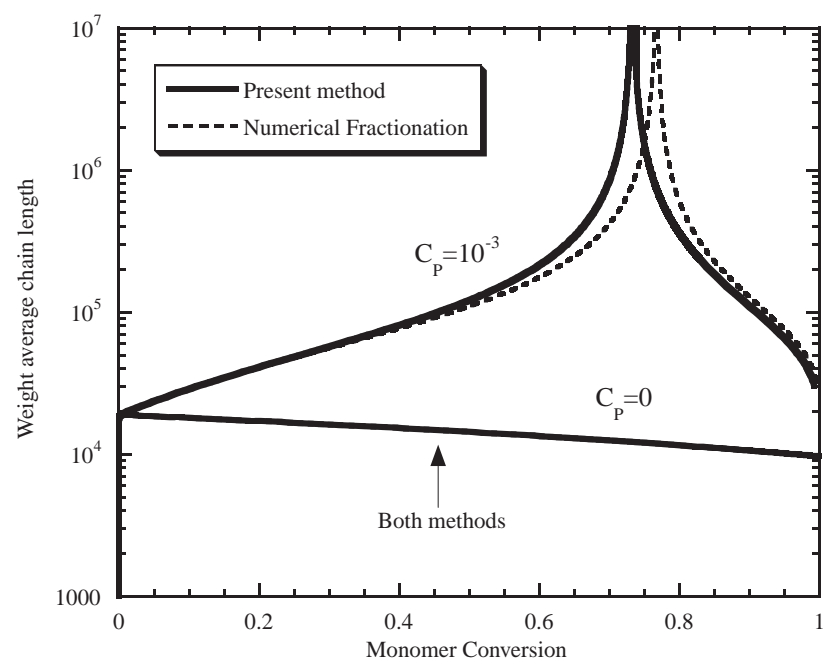

Fig. 11. Effect of transfer to polymer in the predictions of the present method and "Numerical Fractionation" technique for free radical polymerisation with transfer to polymer in a batch reactor.

Although in principle this method should also be able to compute chain length distributions, a lot of improvement in the numerical methods for solving the stiff two point bound-

ary value problems and in the inversion method itself (for decreasing the number of required values of moment generating function) is needed. Evaluation of CLD for chain lengths above a few hundreds is for the moment rather expensive in computer time.

\section{Notation}

a

$A_{j}$

$B$

$C_{I}=\frac{k_{I}}{k_{p}}$

$f_{w_{j}}$

$g_{j}^{-}$and $g_{j}^{+}$

$g_{j}^{*}$

$g_{M_{j}}^{-}$and $g_{M_{j}}^{+}$

$g_{j}^{* *-} \quad$ and
$g_{j}^{* *+}$

$g_{I_{j}}^{-}$and $g_{I_{j}}^{+}$

$G(\mathbf{s})$

$G\left(s_{M}\right)$ vector containing the counts of groups of a polymer molecule, in which $a_{j}$ is the number of groups $A_{j}$ belonging to polymer molecules

mole concentration of groups $A_{j}$ overall concentration of double bonds in case study I

ratio of the two average rate constants of initiation and propagation in case study I initial weight distribution of functionalities in case study I, defined as the initial fraction of active groups belonging to monomer $Y_{j}$

indices of groups (with $g_{j}^{-} \leqslant g_{j}^{+}$) causing the $j$ th bimolecular reaction creating links connecting RU; usually written as $[j-]$ and $[j+]$

index of group causing the $j$ th unimolecular reaction; usually written as $[j *]$ indices of a group belonging to a polymer and a group, corresponding to a monomer, which are involved in the $j$ th transfer to monomer reaction; usually written as $[M j-]$ and $[M j+]$

groups (with $g_{j}^{* *-} \leqslant g_{j}^{* *+}$ ) reacting in the $j$ th bimolecular reaction not creating links between RU; usually written as $[j * *-]$ and $[j * *+]$

indices of groups (with $g_{I_{j}}^{-} \leqslant g_{I_{j}}^{+}$) involved in the $j$ th bimolecular initiation reaction; usually written as $[I j-]$ and $[I j+]$

moment generating function of polymer NCLD

moment generating function of the NMMD of polymer species 


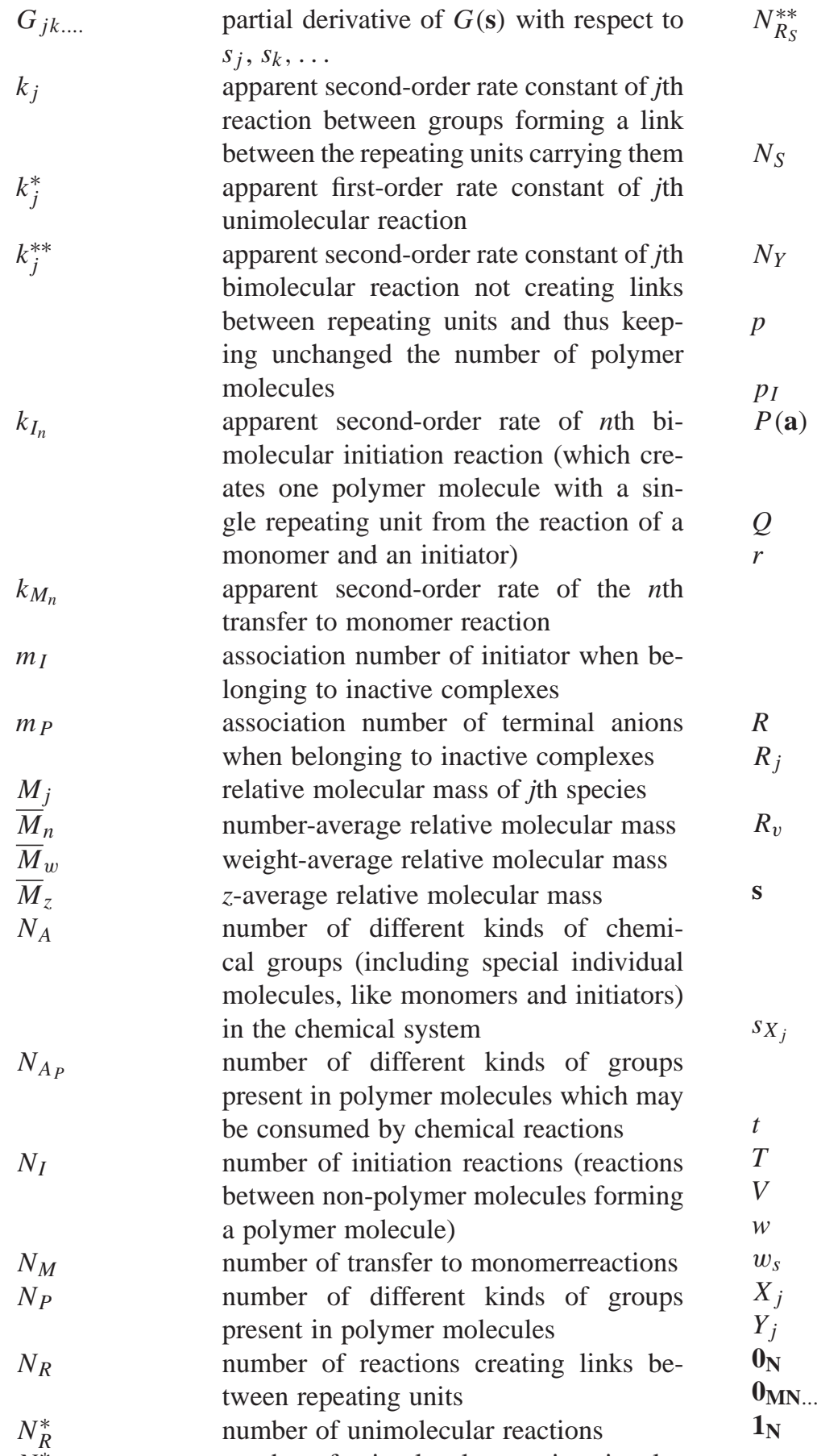

number of unimolecular reactions involving groups in polymer molecules

number of bimolecular reactions which do not create connections between repeating units

$N_{R_{P}}^{* *}$

$N_{R_{P}}$

number of bimolecular reactions involving groups in polymer molecules and which do not create connections between repeating units

number of reactions creating links be-
$N_{R_{S}}^{* *}$

$N_{S}$

$N_{Y}$

$p$

$P(\mathbf{a})$

$Q$

$R_{j}$

$R_{v}$

$X_{j}$

$T$

$w$

$w_{s}$

$X_{j}$

$Y_{j}$

$\mathbf{0}_{\mathrm{MN} \ldots}$

$\mathbf{1}_{\mathbf{N}}$

\section{Greek letters}

$\alpha$

$\gamma_{9}$

$\delta_{A_{j}}$ number of bimolecular reactions involving one group in a polymer molecule and a small molecule, without net creation or destruction of polymer molecules number of bimolecular reactions involving non-polymer molecules without creation of polymer molecules number of different kinds of repeating units overall conversion of double bonds in case study I conversion of initiator in case study I mole concentration of the set of polymer molecules $P(\mathbf{a})$, containing a groups of the various possible kinds volume flow rate

initial ratio of mole concentrations of double bonds and of initiator (same as the final number average degree of polymerisation for a single monofunctional monomer) in case study I

ideal gas constant

rate of formation by chemical reaction of $j$ th species

relative rate of change of volume caused by chemical reaction

vector of Laplace parameters of the moment generating function associated with distributions of the vector a of counts of groups of a polymer species

Laplace parameter of the moment generating function associated with the count of $j$ th $\mathrm{RU}$ of a polymer species time

absolute temperature

reaction volume

weight fraction

weight fraction of sol

mole concentration of $j$ th repeating unit mole concentration of $j$ th monomer

null vector of size $N$

null matrix of size $M \times N \times \cdots$

vector with all its $N$ components equal to one tween RU which involve only groups present in polymer molecules initial mole ratio of divinyl to monovinyl monomer

auxiliary variable in case study I related to $G_{9}$ as defined by Eq. (B.11)

variable which takes the value one when $A_{j}$ is an active group or species which causes chemical reaction and zero when it is only a product of chemical reactions 
$\delta_{P_{j}} \quad$ variable which takes the value one when $A_{j}$ is a group in polymer species and zero when it is an isolated chemical species

$\delta_{j}^{i}$

$\Delta V_{j}$

$\lambda_{0}$

$\lambda_{j k l \ldots}$

$\lambda_{M M}$.

$v_{n k}^{-}$and $v_{n k}^{+}$

$v_{n k}^{*}$

$v_{n k}^{* *-}$
$v_{n k}^{* *+}$$\quad$ and

$v_{I_{n k}}$

$v_{M_{n k}}^{-}$

$v_{M_{n k}}^{+}$

$\Psi_{L}^{U}$

\section{Subscripts}

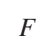

$g$

s

$M A X$

$w$

0

Superscript

Krönecker's symbol, equal to one if $i=j$ and equal to zero otherwise

change of mole volume by the $j$ th chemical reaction

0th order moment of NMMD or NCLD

moment of NMND with respect to the counts of groups $A_{j}, A_{k}, A_{l} \ldots$

$n$th order moment of mole concentration distribution of polymer species with respect to relative molecular mass, in which $n$ is the number of indexes $M$

number of $A_{k}$ groups formed by the $n$th reaction creating connections between $R U$, respectively, in the RU carrying the group with lower index and in the one with upper index

number of $A_{k}$ groups formed by the $n$ thunimolecular reaction

number of $A_{k}$ groups formed by the $n$th bimolecular reaction not creating connections between repeating units, respectively, in the repeating unit carrying the group with lower index and in the one with greater index

number of $A_{k}$ groups of the polymer molecule produced by the $n$th bimolecular initiation reaction (if $k \leqslant N_{A_{P}}$ ), -1 for the two groups starting this reaction change of number of $A_{k}$ groups for the polymer molecule involved in the $n$th transfer to monomer reaction

number of $A_{k}$ groups for the new polymer molecule produced by the $n$th transfer to monomer reaction

space time (ratio of reactor volume and inlet volume flowrate)

stoichiometric functions defined by Eq. (13)

in feed

in gel

number

in sol

upper limit

weight

initial

average of $X$
Abbreviations

$\begin{array}{ll}\text { CLD } & \text { chain length distribution } \\ \text { CSTR } & \text { continuous stirred tank reactor } \\ \text { DVB } & \text { divinylbenzene } \\ \text { EB } & \text { ethylbenzene } \\ \text { LS } & \text { light scattering } \\ \text { NCLD } & \text { number chain length distribution } \\ \text { NMMD } & \text { number molecular mass distribution } \\ \text { RG } & \text { root group (invariant moiety in a RU) } \\ \text { RU } & \text { repeating units } \\ \text { SEC } & \text { size exclusion chromatography }\end{array}$

\section{Acknowledgements}

This research was a part of project PRAXIS XXI and POCTI 3/3.1/CEG/2525/95. Its financial support by FCT, PRODEP, and European Community through FEDER is gratefully acknowledged.

\section{Appendix A}

\section{A.1. Prediction of NCLD and its moments in ideal reactors using the method of characteristics in a CSTR}

The moment generating function of polymer NCLD is obtained by solving the system of ordinary differential equations consisting of Eqs. (A.1)-(A.6) below ( $\delta_{j}^{i}$ is Krönecker's symbol):

$$
\begin{aligned}
\frac{\mathrm{d} G}{\mathrm{~d} t}= & -\sum_{m=1}^{N_{R_{P}}} k_{m} \Psi_{m}^{+} \Psi_{m}^{-} G_{[m-]} G_{[m+]} \\
& +\sum_{m=1}^{N_{I}} k_{I_{m}} A_{[I m-]} A_{[I m+]} \Psi_{I_{m}} \\
& +\sum_{m=1}^{N_{M}} k_{M_{m}} A_{[M m-]} A_{[M m+]} \Psi_{M_{m}}^{+} \\
& +\frac{G_{F}(t)-G(t)}{\tau}-R_{v} G,
\end{aligned}
$$

$$
\begin{aligned}
\frac{\mathrm{d} G_{j}}{\mathrm{~d} t}= & \sum_{m=1}^{N_{R_{P}}} k_{m}\left(v_{m j}^{-}+v_{m j}^{+}\right) \Psi_{m}^{-} \Psi_{m}^{+} G_{[m-]} G_{[m+]} \\
& +\sum_{m=N_{R_{P}}+1}^{N_{R}} k_{m}\left(v_{m j}^{-}+v_{m j}^{+}\right) \Psi_{m}^{-} \Psi_{m}^{+} A_{[m+]} G_{[m-]} \\
& +\sum_{m=1}^{N_{R_{P}}^{*}} k_{m}^{*} v_{m j}^{*} \Psi_{m}^{*} G_{[m *]}
\end{aligned}
$$




$$
\begin{aligned}
& \sum_{m=1}^{N_{R_{P}}^{* *}} k_{m}^{* *}\left(v_{m j}^{* *-} \Psi_{m}^{* *-} A_{[m * *+]} G_{[m * *-]}\right. \\
+ & \left.v_{m j}^{* *+} \Psi_{m}^{* *+} A_{[m * *-]} G_{[m * *+]}\right) \\
+ & \sum_{m=N_{R_{P}}^{*}+1}^{N_{R}^{* *}-N_{S}} k_{m}^{* *} v_{m j}^{* *-} \Psi_{m}^{* *-} A_{[m * *+]} G_{[m * *-]} \\
+ & \sum_{m=1}^{N_{I}} k_{I_{m}} v_{I_{m j}} \Psi_{I_{m}} A_{[I m-]} A_{[I m+]} \\
+ & \sum_{m=1}^{N_{m}} k_{M_{m}} A_{[M m+]}\left(v_{M_{m j}}^{+} \Psi_{M_{m}}^{+} A_{[M m-]}\right. \\
+ & \left.v_{M_{m j}}^{-} \Psi_{M_{m}}^{-} G_{[M m-]}\right) \\
+ & \frac{G_{j_{F}}(t)-G_{j}}{\tau}-R_{v} G_{j},
\end{aligned}
$$

$$
\begin{aligned}
& \frac{\mathrm{d} \log s_{j}}{\mathrm{~d} t}=\sum_{m=1}^{N_{R_{P}}} k_{m}\left[\delta_{[m-]}^{j}\left(A_{[m+]}-\Psi_{m}^{-} \Psi_{m}^{+} G_{[m+]}\right)\right. \\
& \left.+\delta_{[m+]}^{j}\left(A_{[m-]}-\Psi_{m}^{-} \Psi_{m}^{+} G_{[m-]}\right)\right] \\
& +\sum_{m=N_{R_{P}}+1}^{N_{R}} k_{m} \delta_{[m-]}^{j} A_{[m+]}\left(1-\Psi_{m}^{-} \Psi_{m}^{+}\right) \\
& +\sum_{m=1}^{N_{R_{P}}^{*}} k_{m}^{*} \delta_{[m *]}^{j}\left(1-\Psi_{m}^{*}\right) \\
& +\sum_{m=1}^{N_{R_{P}}^{* *}} k_{m}^{* *}\left[\delta_{[m * *-]}^{j} A_{[m * *+]}\left(1-\Psi_{m}^{* *-}\right)\right. \\
& \left.+\delta_{[m * *+]}^{j} A_{[m * *-]}\left(1-\Psi_{m}^{* *+}\right)\right] \\
& N_{R}^{* *}-N_{S} \\
& +\sum_{m=N_{R_{P}}^{*}+1}^{N_{R}^{* *}-N_{S}} k_{m}^{* *} \delta_{[m * *-]}^{j} A_{[m * *+]}\left(1-\Psi_{m}^{* *-}\right) \\
& +\sum_{m=1}^{N_{m}} k_{M_{m}} \delta_{[M m-]}^{j} A_{[M m+]}\left(1-\Psi_{M_{m}}^{-}\right),
\end{aligned}
$$

$G_{\mid t=0}=G_{0}\left(\mathbf{s}_{0}\right)$,

$G_{j_{\mid t=0}}=\frac{\partial G_{0}}{\partial \log s_{j}}\left(\mathbf{s}_{0}\right)$,

$s_{j \mid t=0}=s_{j_{0}}$

Vector $\mathbf{s}_{0}$ defining the starting point of the characteristic at time $t=0$ must be computed in order that the characteristic curve passes through the prescribed values of $\mathbf{s}$ at time $t$. In fact, only the $N_{A_{P}}$ components related to the active polymer groups are unknown, since the other are constant values. Therefore, a two-point boundary value problem for the above system of ordinary differential equations must be solved.

In the prediction of average molecular weights, only the solution at $\mathbf{s}=1_{N_{A}}$ is wanted and the problem becomes much simpler if there is no gelation, because the solution is then $\mathbf{s}_{0}=1_{N_{A}}$ and there is no need to solve the above mentioned two point boundary value problem. After computing vector $\mathbf{s}_{0}$ (in case there is gel) or, alternatively, setting it equal to $\mathbf{1}_{N_{A}}$, it becomes possible to obtain $G$ and any of its derivatives at $\mathbf{s}=1_{N_{A}}$, yielding thus the moments of the NCLD of polymer and finally the moments of the NMMD of polymer, leading to weight fraction of sol and average molecular weights.

Differentiation of Eq. (18) leads to additional ordinary differential equations analogous to Eq. (A.2) above, which are used to compute higher positive integer order derivatives of $G$, such as:

$$
\begin{aligned}
& \frac{\mathrm{d} G_{j k}}{\mathrm{~d} t} \\
& =\sum_{m=1}^{N_{R_{P}}} k_{m} \Psi_{m}^{-} \Psi_{m}^{+}\left[\left(v_{m j}^{-}+v_{m j}^{+}\right)\left(v_{m k}^{-}+v_{m k}^{+}\right) G_{[m-]} G_{[m+]}\right. \\
& +\left(v_{m j}^{-}+v_{m j}^{+}\right)\left(G_{[m-] k} G_{[m+]}+G_{[m+] k} G_{[m+]}\right) \\
& +\left(v_{m k}^{-}+v_{m k}^{+}\right)\left(G_{[m-] j} G_{[m+]}+G_{[m+] j} G_{[m-]}\right) \\
& \left.+G_{[m-] j} G_{[m+] k}+G_{[m+] j} G_{[m-] k}\right] \\
& +\sum_{m=N_{R_{P}}+1}^{N_{R}} k_{m} \Psi_{m}^{-} \Psi_{m}^{+} A_{[m+]}\left[\left(v_{m j}^{-}+v_{m j}^{+}\right)\left(v_{m k}^{-}+v_{m k}^{+}\right)\right. \\
& \times G_{[m-]}+\left(v_{m j}^{-}+v_{m j}^{+}\right) G_{[m-] k}+\left(v_{m k}^{-}+v_{m k}^{+}\right) G_{[m-] j]} \\
& +\sum_{m=1}^{N_{R_{P}}^{*}} k_{m}^{*} \Psi_{m}^{*}\left(v_{m k}^{*} G_{[m *] j}\right. \\
& \left.+v_{m j}^{*} G_{[m *] k}+v_{m j}^{*} v_{m k}^{*} G_{[m *]}\right) \\
& +\sum_{m=1}^{N_{R_{P}}^{* *}} k_{m}^{* *}\left[A _ { [ m * * + ] } \Psi _ { m } ^ { * * - } \left(v_{m k}^{* *-} G_{[m * *-] j}\right.\right. \\
& \left.+v_{m j}^{* *-} G_{[m * *-] k}+v_{m j}^{* *-} v_{m k}^{* *-} G_{[m * *+]}\right) \\
& +A_{[m * *-]} \Psi_{m}^{* *+}\left(v_{m k}^{* *+} G_{[m * *+] j}\right. \\
& \left.\left.+v_{m j}^{* *+} G_{[m * *+] k}+v_{m j}^{* *+} v_{m k}^{* *+} G_{[m * *+]}\right)\right] \\
& +\sum_{m=N_{R_{P}}^{*}+1}^{N_{R}^{* *}-N_{S}} k_{m}^{* *} A_{[m * *+]} \Psi_{m}^{* *-}\left(v_{m k}^{* *-} G_{[m * *-] j}\right.
\end{aligned}
$$




$$
\begin{aligned}
& \left.+v_{m j}^{* *-} G_{[m * *-] k}+v_{m j}^{* *-} v_{m k}^{* *-} G_{[m * *-]}\right) \\
& +\sum_{m=1}^{N_{m}} k_{M_{m}} A_{[M m+]}\left[A_{[M m-]} v_{M_{m j}}^{+} v_{M_{m k}}^{+} \Psi_{M_{m}}^{+}\right. \\
& +\Psi_{M_{m}}^{-}\left(v_{M_{m j}}^{-} G_{[M m-] k}+v_{M_{m k}}^{-} G_{[M m-] j}+v_{M_{m j}}^{-} v_{M_{m k}}^{-}\right. \\
& \left.\left.\times G_{[M m-]}\right)\right]+\sum_{m=1}^{N_{I}} k_{I_{m}} A_{[I m-]} A_{[I m+]} v_{I_{m j}} v_{I_{m k}} \Psi_{I_{m}} \\
& +\frac{G_{j k_{F}}(t)-G_{j k}}{\tau}-R_{v} G_{j k} .
\end{aligned}
$$

Numerical solution of those systems of ordinary differential equations requires the jacobian of their right-hand sides, which can be analytically obtained. It is not presented here by conciseness, but is available free of charge from the authors.

No gel exists if there is no second solution for $\mathbf{s}_{0}$ when $\mathbf{s}=1_{N_{A}}$ with real positive values lower than 1 for any of its components; if it does exist, that solution should be chosen instead of $\mathbf{s}_{0}=1_{N_{A}}$.

In practice, it is better to compute gel time $t_{g}$ as the time for which weight average degree of polymerisation becomes infinite and check afterwards whether the reaction time is higher than $t_{g}$.

\section{Appendix B}

\section{B.1. Analytical treatment of a simple non-linear living polymerisation in a batch reactor}

A more elegant way of writing Eq. (20), also leading to a slight generalisation of this kinetic scheme, results when the set of Laplace parameters of RG $s_{X_{j}}\left(j=1, N_{Y}\right)$ is introduced. In this case, $s_{X_{1}}=s_{4}$ and $s_{X_{2}}=s_{8}$. If there are still other monomers, provided their functional groups have the same reactivities, in the above scheme, assuming that monomer $Y_{j}$ has functionality $j$, Eq. (20) may be rewritten as:

$$
\begin{aligned}
\frac{\partial G}{\partial t}= & k_{I} I\left[s_{2} s_{5} \sum_{j=1}^{N_{Y}} j s_{X_{j}} s_{9}^{j-1} Y_{j}+\frac{\partial G}{\partial \log s_{9}}\left(\frac{s_{2} s_{5}}{s_{9}}-1\right)\right] \\
& +k_{p}\left\{\frac { \partial G } { \partial \operatorname { l o g } s _ { 5 } } \left[\sum_{j=1}^{N_{Y}} j Y_{j}\left(s_{6} s_{X_{j}} s_{9}^{j-1}-1\right)\right.\right. \\
& \left.\left.+\frac{s_{6}}{s_{9}} \frac{\partial G}{\partial \log s_{9}}-A_{9}\right]-A_{5} \frac{\partial G}{\partial \log s_{9}}\right\}
\end{aligned}
$$

The equations of the characteristics derived from Eq. (B.1) are:

$$
\begin{aligned}
& \frac{\mathrm{d} G}{\mathrm{~d} t}= k_{I} I s_{2} s_{5} \sum_{j=1}^{N_{Y}} j s_{X_{j}} s_{9}^{j-1} Y_{j}+k_{p} \frac{s_{6}}{s_{9}} G_{5} G_{9}, \\
& \frac{\mathrm{d} G_{5}}{\mathrm{~d} t}= k_{I} I s_{2} s_{5}\left(\sum_{j=1}^{N_{Y}} j s_{X_{j}} s_{9}^{j-1} Y_{j}+\frac{G_{9}}{s_{9}}\right) \\
&= k_{I} I s_{2} s_{5} B \gamma_{9}, \\
& \frac{\mathrm{d} G_{9}}{\mathrm{~d} t}=\left(k_{I} I s_{2} s_{5}+k_{p} s_{6} G_{5}\right) \\
& \times {\left[\sum_{j=1}^{N_{Y}} j(j-1) s_{X_{j}} s_{9}^{j-1} Y_{j}-\frac{G_{9}}{s_{9}}\right], } \\
& \frac{\mathrm{d} \log s_{5}}{\mathrm{~d} t}=k_{p} B\left(1-s_{6} \gamma_{9}\right), \\
& \frac{\mathrm{d} \mathrm{log} s_{9}}{\mathrm{~d} t}=k_{I} I\left(1-\frac{s_{2} s_{5}}{s_{9}}\right)+k_{p}\left(A_{5}-\frac{s_{6} G_{5}}{s_{9}}\right) .
\end{aligned}
$$

Assuming that reaction starts from monomers only, but allowing for a partial conversion of double bonds resulting from instantaneous initiation, the initial conditions are:

$$
\begin{aligned}
s_{j \mid t=0}= & s_{j_{0}} \quad j=5,9, \\
G_{\mid t=0}= & B_{0} \sum_{j=1}^{N_{Y}} \frac{f_{w_{j}}}{j} s_{X_{j}}\left\{\left[s_{2} s_{5_{0}} p_{0}+s_{9_{0}}\left(1-p_{0}\right)\right]^{j}\right. \\
& \left.-\left[s_{9_{0}}\left(1-p_{0}\right)\right]^{j}\right\}, \\
G_{9 \mid t=0}= & B_{0} s_{9_{0}}\left(1-p_{0}\right) \sum_{j=1}^{N_{Y}} f_{w_{j}} s_{X_{j}}\left\{\left[s_{2} s_{5_{0}} p_{0}\right.\right. \\
& \left.\left.+s_{9_{0}}\left(1-p_{0}\right)\right]^{j-1}-\left[s_{9_{0}}\left(1-p_{0}\right)\right]^{j-1}\right\}, \\
G_{5 \mid t=0}= & I_{0} s_{2} s_{5_{0}} \sum_{j=1}^{N_{Y}} f_{w_{j}} s_{X_{j}}\left[s_{2} s_{5_{0}} p_{0}\right. \\
& \left.+s_{9_{0}}\left(1-p_{0}\right)\right]^{j-1}=I_{0} s_{2} s_{5_{0}} \gamma_{0} .
\end{aligned}
$$

Owing to the binomial integral in Eq. (33), a full analytical solution is possible only for constant $C_{I}$, including the case of instantaneous initiation. The crux for obtaining the analytical solution is the invariance along each characteristic of the auxiliary variable $\gamma_{9}$ :

$\gamma_{9}=\frac{G_{9}}{B s_{9}}+\sum_{j=1}^{N_{Y}} s_{X_{j}} s_{9}^{j-1} \frac{j Y_{j}}{B}$.

According to these solutions, the first step to compute $G(\mathbf{s})$ consists in solving a single algebraic equation in terms of the auxiliary variable $\gamma_{9}$, Eqs. (B.12), or (B.13) for instantaneous 
initiation:

$$
\begin{aligned}
\gamma_{9}= & \sum_{j=1}^{N_{Y}} f_{w_{j}} s_{X_{j}}\left\{s_{9}(1-p)+\frac{s_{2} s_{5}}{r}\right. \\
& \times\left\{\frac{C_{I}\left(C_{I}-1\right)\left[1-\left(1-p_{I}\right)^{\frac{s_{6} \gamma_{9}+C_{I}-1}{C_{I}}}\right]}{\left(s_{6} \gamma_{9}+C_{I}-1\right)^{2}}\right. \\
& \left.\left.-\frac{s_{6} \gamma_{9} \log \left(1-p_{I}\right)}{s_{6} \gamma_{9}+C_{I}-1}\right\}\left(1-p_{I}\right)^{\frac{1-s_{6} \gamma_{9}}{C_{I}}}\right\}^{j-1},
\end{aligned}
$$

$$
\begin{aligned}
\gamma_{9}= & \sum_{j=1}^{N_{Y}} f_{w_{j}} s_{X_{j}}\left\{s_{9}(1-p)+s_{2} s_{5}\left[\frac{1}{r}+s_{6} \gamma_{9}\left(p-\frac{1}{r}\right)\right]\right. \\
& \left.\times \exp \left[\left(1-s_{6} \gamma_{9}\right)(1-r p)\right]\right\}^{j-1} \quad C_{I} \rightarrow \infty, \quad \text { B. } 13
\end{aligned}
$$

$s_{5_{0}}=s_{5}\left(1-p_{I}\right)^{\frac{1-s_{6} \gamma_{9}}{C_{I}}}$,

$$
G_{5}=\frac{I_{0} C_{I} \gamma_{9} s_{2} s_{5_{0}}\left[1-\left(1-p_{I}\right)^{\frac{s_{6} \gamma_{9}+C_{I}-1}{C_{I}}}\right]}{s_{6} \gamma_{9}+C_{I}-1},
$$

$$
\begin{aligned}
s_{9}(1-p)= & s_{9_{0}}+\frac{s_{2} s_{5_{0}}}{r}\left\{\frac{s_{6} \gamma_{9} \log \left(1-p_{I}\right)}{C_{I}-1+s_{6} \gamma_{9}}\right. \\
& +\frac{C_{I}\left(C_{I}-1\right)}{\left(s_{6} \gamma_{9}+C_{I}-1\right)^{2}} \\
& \left.\times\left[1-\left(1-p_{I}\right)^{\frac{C_{I}-1+s_{6} \gamma_{9}}{C_{I}}}\right]\right\},
\end{aligned}
$$

$\frac{G_{9}}{B_{0}}=\gamma_{9} s_{9}(1-p)-\sum_{j=1}^{N_{Y}} f_{w_{j}} s_{X_{j}}\left[s_{9}(1-p)\right]^{j}$,

$$
\begin{aligned}
\frac{G}{B_{0}}= & \frac{C_{I} s_{2} s_{6} s_{5_{0}} \gamma_{9}^{2}}{s_{6} \gamma_{9}+C_{I}-1} \\
& \times\left[\frac{1-\left(1-p_{I}\right)^{\frac{C_{I}-1+s_{6} \gamma_{9}}{C_{I}}}}{r\left(s_{6} \gamma_{9}+C_{I}-1\right)}-p+\frac{\left(C_{I}-1\right) p_{I}}{C_{I} r}\right] \\
& +\sum_{j=1}^{N_{Y}} \frac{f_{w_{j}}}{j} s_{X_{j}}\left\{s_{9_{0}}^{j}-\left[s_{9}(1-p)\right]^{j}\right\}
\end{aligned}
$$

For instantaneous initiation, the simplified relationships below hold:

$$
\begin{aligned}
s_{5_{0}} & =s_{5} \exp \left[\left(1-s_{6} \gamma_{9}\right)(1-r p)\right], \\
G_{5} & =\frac{B_{0}}{r} s_{2} s_{5_{0}} \gamma_{9}, \\
s_{9_{0}} & =\frac{s_{9}(1-p)}{1-1 / r}+\frac{s_{2} s_{6} s_{5_{0}} \gamma_{9}(r p-1)}{r-1}, \\
\frac{G_{9}}{B_{0}} & =\gamma_{9} s_{9}(1-p)-\sum_{j=1}^{N_{Y}} f_{w_{j}} s_{X_{j}}\left[s_{9}(1-p)\right]^{j},
\end{aligned}
$$

$$
\begin{aligned}
\frac{G}{B_{0}}= & \gamma_{9}\left[s_{9}(1-p)-s_{9_{0}}(1-1 / r)\right]+\sum_{j=1}^{N_{Y}} \frac{f_{w_{j}}}{j} s_{X_{j}} \\
& \times\left\{\left[s_{9_{0}}(1-1 / r)\right]^{j}-\left[s_{9}(1-p)\right]^{j}\right\} .
\end{aligned}
$$

Eqs. (B.12) or (B.13) have multiple solution branches. The physically meaningful branch has to be chosen from the behaviour at $\mathbf{s}=1_{N_{A}}$.

From its definition Eq. (B.11), taking into account the meaning of $G_{9}$ as well as Eqs. (B.17) or (B.18), $\gamma_{9}$ is a real positive value less than or equal to 1 . If all polymer molecules are finite, $\gamma_{9}=1$; this solution always exists. If a second solution for $\gamma_{9}$ between 0 and 1 can be found, it takes precedence over that trivial solution $\gamma_{9}=1$, and this means that an infinite network is present.

Stating that a double root 1 exists for Eqs. (B.12) or (B.13), Eq. (34) for critical gel conversions is obtained. Differentiation of equations above on $\mathbf{s}=1_{N_{A}}$ would allow analytical expressions for the various moments of the NCLD to be obtained.

It is possible to obtain a full analytical solution (Tobita, 1994a) for a somewhat simpler description (only mono- and difunctional monomers were considered in that work). It is useful for testing numerical inversion methods.

\section{References}

Abate, J., Whitt, W., 1992. Numerical inversion of probability generating functions. Operations Research Letters 12, 245-251.

Achilias, D.S., Kiparissides, C., 1992. Toward the development of a general framework for modelling molecular weight and compositional changes in free-radical copolymerisation reactions. Journal of Macromolecular Science, Reviews in Macromolecular Chemistry Phys. C 32, 183-234.

Arest-Yakubovich, A.A., 1997. On the kinetics of lithium-initiated anionic polymerization in nonpolar solvents. Journal of Polymer Science Part A: Polymer Chemistry Edition 35, 3613-3615.

Asteasuain, M., Sarmoria, C., Brandolin, A., 2002a. Peroxide modification of polyethylene. Prediction of molecular weight distributions by probability generating functions. Polymer 43, 2363-2373.

Asteasuain, M., Sarmoria, C., Brandolin, A., 2002b. Recovery of molecular weight distributions from transformed domains. Part I. Application of pgf to mass balances describing reactions involving radicals. Polymer 43, 2513-2527.

Asteasuain, M., Sarmoria, C., Brandolin, A., 2004. Molecular weight distributions in styrene polymerisation with asymmetric difunctional initiators. Polymer 45, 321-335.

Bamford, C.H., Tompa, H., 1954. The calculation of molecular weight distributions from kinetic schemes. Transactions of the Faraday Society 50, 1097-1115.

Bashir-Ali, Z., Cash, J.R., Silva, H.H.M., 1998. Lobatto deferred correction for stiff two-point boundary value problems. Comput. Math. Appl. 36, 59-69.

Brandolin, A., Sarmoria, C., Lòpez-Rodríguez, A., Whiteley, K.S., del Amo Fernández, B., 2002. Prediction of molecular weight distributions by probability generating functions. Application to industrial autoclave reactors for high pressure polymerisation of ethylene and ethylenevinyl acetate. Polymer Engineering and Science 41, 1413-1426.

Bywater, S.D., 1998. Active centre aggregation in lithium-based anionic polymerization. Are very large aggregates present? Macromolecules 31, 6010-6013. 
Bywater, S.D., Worsfold, J., 1967. Alkyl lithium anionic polymerisation initiators in hydrocarbon solvents. Journal of Organometallic Chemistry $10,1-6$.

Cash, J.R., Moore, G., Wright, R.W., 1995. An automatic continuation strategy for the solution of singularly perturbed linear two point boundary value problems. Journal of Computational Physics 122, 266-279.

Cash, J.R., Moore, G., Wright, R.W., 2001. An automatic continuation strategy for the solution of singularly perturbed nonlinear boundary value problems. ACM Transactions on Mathematical Software 27, 245-266.

Christiansen, W.H., Ekerdt, J.G., Trachtenberg, I., Barlow, J.W., 1990. Development of a reaction injection molding encapsulant system. 2. Chemorheology of the anionic bulk polymerization of styrene. Industrial and Engineering Chemistry Research 30, 646-653.

Costa, M.R.P.F.N., Dias, R.C.S., 1994. A general kinetic analysis of nonlinear irreversible copolymerisations. Chemical Engineering Science 49, 491-516.

Costa, M.R.P.F.N., Dias, R.C.S., 1995. New developments in the kinetic modelling of complex non-linear polymerizations. In: Reichert, K.H., Moritz, H.-U. (Eds.), Fifth International Workshop on Polymer Reaction Engineering, DECHEMA Monographs, vol. 131. Wiley-VCH, Weinheim, pp. 523-530.

Costa, M.R.P.F.N., Dias, R.C.S., 1998. Prediction of average radius of gyration of branched polymers by a general kinetic approach. In: Reichert, K.H., Moritz, H.-U. (Eds.), Sixth International Workshop on Polymer Reaction Engineering, DECHEMA Monographs, vol. 134. Wiley-VCH, Weinheim, pp. 197-205.

Costa, M.R.P.F.N., Dias, R.C.S., 2003. Prediction of sol fraction and average molecular weights after gelation for non-linear free radical polymerizations using a kinetic approach. Macromolecular Theory and Simulations 12, 560-572.

Costa, M.R.P.F.N., Villermaux, J., 1988. Mathematical and experimental foundations of linear polycondensation modeling. 1. Modeling and simulation of linear, irreversible polycondensation. Industrial and Engineering Chemistry Research 27, 421-429.

Courant, R., Hilbert, D., 1962. Methods of Mathematical Physics, Vol. II-Partial Differential Equations. Wiley-Interscience, New York, pp. 97-105.

Deuflhard, P., Bornemann, F., 2002. Scientific Computing with Ordinary Differential Equations. Springer, Berlin, pp. 390.

Elliott, J.B., Bowman, C.N., 2002. Effect of primary cyclization on free radical polymerization kinetics: modeling approach. Macromolecules 35, 7125-7131.

Eschwey, H., Burchard, W., 1975. Conditions of gelation in the anionic divinylbenzene-styrene copolymerisation. Journal of Polymer Science, Polymer Symposia Series 53, 1-9.

Flory, P.J., 1953. Principles of Polymer Chemistry, Cornell University Press, Ithaka, NY. (Chapter 9).

Hairer, E., Wanner, G., 2002. Solving Ordinary Differential Equations II, second revised ed. Springer, Berlin, pp. 566-574.

Hayman, W., 1956. A generalisation of Stirling's formula. Journal für die Reine and Angewandte Mathematik 196, 67-195.

Hild, G., Okasha, R., 1985a. Kinetic investigation of the free-radical crosslinking copolymerization in the pre-gel state. 1 . Styrene $/ m$ - and p-divinylbenzene systems. Makromolekulare Chemie 186, 93-110.

Hild, G., Okasha, R., 1985b. Free radical crosslinking copolymerization in the pre-gel state, 2. Styrene/ethylene dimethacrylate and styrenediisopropenylbenzene systems. Makromolekulare Chemie 186, 389-406.

Hild, G., Okasha, R., Rempp, P., 1985. Free radical crosslinking copolymerization in the post-gel state, 3. Swelling and mechanical properties of polystyrene networks. Makromolekulare Chemie 186, 407-422.

Hutchinson, K.A., Richards, J.R., Aronson, M.T., 1994. Determination of propagation rate coefficients by pulsed-laser polymerization for systems with rapid chain growth: vinyl acetate. Macromolecules 27, 4530-4537.

Iedema, P.D., Hoefsloot, H.C., 2002. Molecular-weight-distribution modelling of radical polymerization in batch and continuous reactors with transfer to polymer leading to gel formation. Macromolecular Theory and Simulations 11, 410-428.

Karles, G.D., Christiansen, W.H., Ekerdt, J.G., Trachtenberg, I., Barlow, J.W., 1991. Development of a reaction injection molding encapsulant system. 3. Gel times for the anionic bulk polymerization of styrene with divinylbenzene. Industrial and Engineering Chemistry Research 30, 646-653.

Keller, H.B., 1972. Numerical Methods for Two-Point Boundary-Value Problems, second ed. Dover, New York, pp. 61.

Kern, W.J., Anderson, J.N., Adams, H.E., Bouton, T.C., Bethea, T.W., 1972. Thermal stability of living polymer-lithium systems. Journal of Polymer Science Part A: Polymer Chemistry Edition 16, 3123-3131.

Konstadinides, K., Achilias, D.S., Kiparissides, C., 1992. Development of a unified mathematical framework for modeling molecular and structural changes in free-radical homopolymerisation reactions. Polymer 33, 5019-5031.

Lewis, H.L., Brown, T.L., 1970. Association of alkyllithium compounds in hydrocarbon media. Alkyllithium-base interactions. Journal of the American Chemical Society 92, 4664-4670.

Mikeš, J., Dušek, K., 1982. Simulation of polymer network formation by the Monte Carlo method. Macromolecules 15, 93-99.

Miller, N.C., Toffolo, R.W., McAuley, K.B., McLellan, P.J., 1996. Determining polymer chain length distributions using numerical inversion of Laplace transforms. Polymer Reaction Engineering 4, 279-301.

Mills, P.L., 1986. Determination of polymer chain length distributions by numerical inversion of $z$-transforms. Computers \& Chemical Engineering 10, 399-420.

Priddy, D.B., Pirc, M., Meister, B.J., 1992. Development of continuous anionic styrene polymerization technology. Polymer Reaction Engineering 1, 343-356.

Ray, W.H., 1991. Modeling of addition polymerisation processes-free radical, ionic, group transfer and Ziegler-Natta kinetics. Canadian Journal of Chemical Engineering 69, 626-629.

Saldivar, E., Ray, W.H., 1995. Detailed modeling of emulsion copolymerisation processes. In: Reichert, K.H., Moritz, H.-U. (Eds.), Fifth International Workshop on Polymer Reaction Engineering, DECHEMA Monographs, vol. 131. VCH, Weinheim, pp. 105-112.

Spach, G., Levy, M., Szwarc, M., 1962. Spontaneous slow transformations in the "living" polystyrene system. Journal of the Chemical Society, 355-361.

Teymour, F., Campbell, J.D., 1994. Analysis of the dynamics of gelation in polymerization reactors using the "numerical fractionation" technique. Macromolecules 27, 2460-2469.

Tobita, H., 1994a. Dimensions of cross-linked polymers formed in living vinyl/divinyl copolymerization. Macromolecules 27, 5413-5420.

Tobita, H., 1994b. A simulation model for long-chain branching in vinyl acetate polymerization: 1. Batch polymerization. Journal of Polymer Science Part B: Polymer Physics Edition 32(B), 901-910.

Tobita, H., 1994c. A simulation model for long-chain branching in vinyl acetate polymerization: 2 . Continuous polymerization in a stirred tank reactor. Journal of Polymer Science Part B: Polymer Physics Edition 32(B), 911-919.

Tobita, H., Hamielec, A.E., 1988. A kinetic model for network formation in free radical polymerization. Makromolekulare Chemie, Macromolecular Symposia 20/21, 501-543.

Tobita, H., Hamielec, A.E., 1989. Crosslinking kinetics in free radical copolymerization. In: Reichert, K.H., Geiseler, W. (Eds.), Proceedings of the Third International Workshop on Polymer Reaction Engineering. VCH, Weinheim, pp. 43-83.

Tobita, H., Zhu, S., 1996. Polyradical distribution in free radical crosslinking of polymer chains. Journal of Polymer Science Part B: Polymer Physics Edition 34(B), 2099-2104. 
Villermaux, J., Blavier, L., 1984. Free radical polymerisation engineering-I: A new method for modeling free radical homogeneous polymerisation reactions. Chemical Engineering Science 39, 87-99.

Worsfold, D.J., 1970. Anionic polymerization of styrene with paradivinylbenzene. Macromolecules 3, 514-517.

Worsfold, D.J., Bywater, S., 1960. Anionic polymerization of styrene. Canadian Journal of Chemistry 38, 1891-1900.

Young, R.N., Quirk, R.P., Fetters, L.J., 1984. Anionic polymerizations of non-polar monomers involving lithium. Advances in Polymer Science $56,1-90$.
Your, J.-J.A., Karles, G.D., Ekerdt, J.G., Trachtenberg, I., Barlow, J.W., 1989. Development of a reaction injection molding encapsulant system. 1. Kinetic studies of a butyllithium catalyzed styrene polymerization. Industrial and Engineering Chemistry Research 28, 1456-1463.

Zetterlund, P.B., Yamazoe, H., Yamada, B., 2002. Propagation and termination kinetics in high conversion free radical co-polymerization of styrene/divinylbenzene investigated by electron-spin resonance and Fourier transform near-infrared spectroscopy. Polymer 43, 7027-7035. 